\title{
Random Polytopes, Convex Bodies, and Approximation
}

\author{
Imre Bárány \\ Rényi Institute of Mathematics \\ H-1364 Budapest Pf. 127 Hungary \\ e-mail: barany@renyi.hu \\ and \\ Mathematics, University College London \\ Gower Street, London, WC1E 6BT, UK
}

Assume $K \subset \mathbb{R}^{d}$ is a convex body and $X_{n} \subset K$ is a random sample of $n$ uniform, independent points from $K$. The convex hull of $X_{n}$ is a convex polytope $K_{n}$ called random polytope inscribed in $K$. We are going to investigate various properties of this polytope: for instance how well it approximates $K$, or how many vertices and facets it has. It turns out that $K_{n}$ is very close to the so called floating body inscribed in $K$ with parameter $1 / n$. To show this we develop and use the technique of cap coverings and Macbeath regions. Its power will be illustrated, besides random polytopes, on several examples: floating bodies, lattice polytopes, and approximation problems.

\section{Introduction}

We write $\mathcal{K}$ or $\mathcal{K}^{d}$ for the set of convex bodies in $\mathbb{R}^{d}$, that is, compact convex sets with nonempty interior in $\mathbb{R}^{d}$. Assume $K \in \mathcal{K}$ and $x_{1}, \ldots, x_{n}$ are random, independent points chosen according to the uniform distribution in $K$. The convex hull of these points, to be denoted by $K_{n}$, is called a random polytope inscribed in $K$. Thus $K_{n}=\left[x_{1}, \ldots, x_{n}\right]$ where $[S]$ stands for the convex hull of the set $S$. The study of random polytopes began with Sylvester's famous "four-point question" [55]. For more information and recent results on the four-point question see [7] and [8].

Starting with the work of Rényi and Sulanke [39] there has been a lot of research to understand the asymptotic behaviour of random polytopes. Most of it has been concentrated on the expectation of various functionals associated with $K_{n}$. For instance the number of vertices, $f_{0}\left(K_{n}\right)$, or more generally, the

* Partially supported by Hungarian National Science Foundation Grants T 037846 and T 046246 
number of $k$-dimensional faces, $f_{k}\left(K_{n}\right)$, of $K_{n}$, or the volume missed by $K_{n}$, that is $\operatorname{vol}\left(K \backslash K_{n}\right)$. The latter quantity measures how well $K_{n}$ approximates $K$. As usual we will denote the expectation of $f_{k}\left(K_{n}\right)$ by $\mathbb{E} f_{k}\left(K_{n}\right)$, and that of $\operatorname{vol}\left(K \backslash K_{n}\right)$ by $\mathbb{E}(K, n)$.

In their 1963 paper [39] Rényi and Sulanke made a surprising discovery. Already in the planar case, the expectation of the number of vertices, $f_{0}\left(K_{n}\right)$, depends heavily on the boundary structure of $K$. It is of order $\ln n$ when $K$ is a convex polygon, and is of order $n^{1 / 3}$ when $K$ is a circle (or any other smooth enough convex body). Similarly, $\mathbb{E}(K, n)$ is of order $n^{-2 / 3}$ for smooth convex bodies in $\mathbb{R}^{2}$ and $(\ln n) / n$ for convex polygons. What is the reason for such a different behaviour?

The aim of this survey is to give a thorough introduction to the theory of random polytopes. There are two kinds of results concerning $\mathbb{E}(K, n)$ and $\mathbb{E} f_{k}\left(K_{n}\right)$ : precise asymptotic and order of magnitude. We mainly focus on the second type of results and only mention some precise asymptotics. Along the way we will see why such a different behaviour of $\mathbb{E}(K, n)$ is quite natural. We will introduce the notion of caps, $M$-regions, and cap coverings of convex bodies. They constitute a method to handle the boundary structure of convex bodies. The technique of $M$-regions and cap coverings can be used for other problems as well: several applications will be presented, some of them coming from my paper [6]. This survey contains very little new material. I will indicate at the end of some sections where the results come from and mention if they are new.

I am not quite sure I organized the material of the survey in a concise way. There were too many directions to talk about: random polytopes, $M$ regions, cap coverings, technical preparations and lemmas, probabilistic tools, and further applications. It is difficult to order them linearly (as a survey should be written). Here is the contents, section by section:

1. Introduction

2. Computing $\mathbb{E} \phi\left(K_{n}\right)$

3. Minimal caps and a general result

4. The volume of the wet part

5 . The economic cap covering theorem

6. Macbeath regions

7. Proofs of the properties of Macbeath regions

8. Proof of the cap covering theorem

9. Auxiliary lemmas from probability

10. Proof of Theorem 3.1

11. Proof of Theorem 4.1

12. Proof of (4)

13. Expectation of $f_{k}\left(K_{n}\right)$

14. Proof of Lemma 13.2

15. Further results

16. Lattice polytopes 


\section{Approximation}

18. How it all began: segments on bd $K$

The next section presents a sketch of the method for computing expectations directly. The main results for random polytopes are contained in Section 3. Notation and terminology, including the wet part and the floating body are introduced there. Important properties of the wet part are given in Section 4. The economic cap covering theorem, together with a corollary, is stated next. Macbeath regions are defined and their properties stated in Section 6 . The proofs of these results are given in Sections 7 to 12. Some of them can be skipped on first reading, although the proofs use concepts and methods from proofs from previous sections. We treat separately the expectation of the number of $k$-dimensional faces of $K_{n}$ in Section 13 and 14. This proof is new, using the cap covering technique in a slightly different way and avoiding the probabilistic tools. Further results, including some spectacular new theorems, are explained without proof in Section 15. Applications of the cap-covering technique are given in Sections 16 and 17. The final section is devoted to the origins of the method.

\section{Computing $\mathbb{E} \phi\left(K_{n}\right)$}

The method for computing $\mathbb{E} \phi\left(K_{n}\right)$ goes back to the 1963 paper of Rényi and Sulanke [39] and is the following. Let $P$ be a polytope and write $\mathcal{F}$ for the set of facets of $P$. Assume the function $\phi$ is of the form

$$
\phi(P)=\sum_{F \in \mathcal{F}} \phi(F)
$$

Such functions are $f_{d-1}$, volume, or surface area. The orientation of the facet is given by the outer normal to $P$ at $F$. As $K_{n}$ is a simplicial polytope with probability one, each facet is of the form $\left[x_{i_{1}}, \ldots, x_{i_{d}}\right]$. We write $\mathbf{1}\{E\}$ for the indicator function of the event $E$. Then, assuming vol $K=1$,

$$
\begin{aligned}
\mathbb{E} \phi\left(K_{n}\right)= & \left.\sum_{1 \leq i_{1}<\cdots<i_{d} \leq n} \int_{K} \ldots \int_{K} \mathbf{1}\left\{\left[x_{i_{1}}, \ldots, x_{i_{d}}\right] \in \mathcal{F}\right]\right\} \times \\
& \times \phi\left(\left[x_{i_{1}}, \ldots, x_{i_{d}}\right]\right) \mathrm{d} x_{1} \ldots \mathrm{d} x_{n} \\
= & \left(\begin{array}{l}
n \\
d
\end{array}\right) \int_{K} \ldots \int_{K} 1\left\{\left[x_{1}, \ldots, x_{d}\right] \in \mathcal{F}\right\} \phi\left(\left[x_{1}, \ldots, x_{d}\right]\right) \mathrm{d} x_{1} \ldots \mathrm{d} x_{n} .
\end{aligned}
$$

We will denote by $V=V\left(x_{1}, \ldots, x_{d}\right)$ the volume of the smaller cap cut off from $K$ by aff $\left\{x_{1}, \ldots, x_{d}\right\}$ (which is a hyperplane, almost surely). Here, we used aff $S$ for the affine hull of $S$. Since $F=\left[x_{1}, \ldots, x_{d}\right]$ is a facet, if and only if $x_{d+1}, \ldots, x_{n}$ are all on one side of aff $\left\{x_{1}, \ldots, x_{d}\right\}$, we have the following theorem. 
Theorem 2.1. Under the above conditions,

$$
\mathbb{E} \phi\left(K_{n}\right)=\left(\begin{array}{l}
n \\
d
\end{array}\right) \int_{K} \ldots \int_{K}\left[(1-V)^{n-d}+V^{n-d}\right] \phi(F) \mathrm{d} x_{1} \ldots \mathrm{d} x_{d} .
$$

One can give precise estimates for this integral in several special cases. For instance if $\phi=f_{d-1}$, then $\phi(F)=1$ and the above formula can be directly evaluated for smooth convex sets and for polygons in the plane. In [39], Rényi and Sulanke prove that, for smooth convex sets of area one,

$$
\mathbb{E} f_{1}\left(K_{n}\right)=\left(\frac{2}{3}\right)^{1 / 3} \Gamma\left(\frac{5}{3}\right)\left(\int_{\mathrm{bd} K} \kappa^{1 / 3} \mathrm{~d} s\right) n^{1 / 3}(1+o(1)),
$$

where $\kappa$ is the curvature and integration is by arc length $\mathrm{d} s$ on the boundary bd $K$ of the convex body. For polygons, direct computation in [39] shows that

$$
\mathbb{E} f_{1}\left(K_{n}\right)=\frac{2}{3} f_{0}(K) \ln n(1+o(1))
$$

Of course, $f_{1}=f_{0}$ in these cases. The computation reveals that, for smooth bodies, the vertices of $K_{n}$ are distributed evenly near bd $K$, the boundary of $K$, while for polygons, they are concentrated near the vertices of the original polygon. This is a first level explanation for the different behaviour of $\mathbb{E} f_{0}\left(K_{n}\right)$.

For smooth convex bodies in higher dimension $\mathbb{E} \phi\left(K_{n}\right)$ can sometimes be evaluated using the Blaschke-Petkantschin [43] integral formula. We will return to this in Section 15.

Remark. Equation (1) was used first by Rényi and Sulanke [39] in the planar case.

\section{Minimal Caps and a General Result}

Recalling that $\mathcal{K}$ denotes the set of all convex bodies in $\mathbb{R}^{d}$, we will write $\mathcal{K}_{1}$ for the set of those $K \in \mathcal{K}$ that have unit volume, vol $K=1$. This is convenient since then the Lebesgue measure and the uniform probability measure on $K \in \mathcal{K}_{1}$ coincide.

Assume $a \in \mathbb{R}^{d}$ is a unit vector and $t \in \mathbb{R}$. Then the halfspace $H=H(a \leq$ $t$ ) is defined as

$$
H(a \leq t)=\left\{x \in \mathbb{R}^{d}: a \cdot x \leq t\right\},
$$

where $a \cdot x$ is the scalar product of $a$ and $x$.

A cap of $K \in \mathcal{K}$ is simply a set of the form $C=K \cap H$ where $H$ is a closed halfspace. The width of the cap, $w(C)$ is the usual width of $C$ in the normal direction of $H$. We define the function $v: K \rightarrow \mathbb{R}$ by

$$
v(x)=\min \{\operatorname{vol}(K \cap H): x \in H \text { and } H \text { is a halfspace }\},
$$


This function is going to play a central role in what follows. The minimal cap belonging to $x \in K$ is a cap $C(x)$ with $x \in C(x)$ and vol $C(x)=v(x)$. The minimal cap $C(x)$ need not be unique, so our notation is a little ambiguous but this will not cause any trouble.

The level sets of $v$ are defined as

$$
K(v \geq t)=\{x \in K: v(x) \geq t\} .
$$

The wet part of $K$ with parameter $t>0$ is

$$
K(t)=K(v \leq t)=\{x \in K: v(x) \leq t\} .
$$

The name comes from the mental picture when $K$ is a three dimensional convex body containing $t$ units of water. We call $K(v \geq t)$ the floating body of $K$ with parameter $t>0$ as, in a similar picture, this is the part of $K$ that floats above water (cf. [10] and [32]). The floating body is the intersection of halfspaces, so it is convex.

The general behaviour of $\mathbb{E}(K, n)$ was described in $[10]: \mathbb{E}(K, n)$ is of the same order of magnitude as the volume of the wet part with $t=1 / n$. This works for general convex bodies $K \in \mathcal{K}$, not only when $K$ is smooth or is a polytope. Precisely, we have the following result.

Theorem 3.1. For every $d \geq 2$ there are constants $c_{0}, c_{1}, c_{2}>0$ such that for every $K \in \mathcal{K}_{1}$ and $n \geq c_{0}$

$$
c_{1} \operatorname{vol} K(1 / n) \leq \mathbb{E}(K, n) \leq c_{2} \operatorname{vol} K(1 / n) .
$$

It will be convenient to use the $\ll, \gg$ and $\approx$ notation. For instance, $f(n) \ll$ $g(n)$ means that there is a constant $b$ such that $f(n) \leq b g(n)$ for all values of $n$. This notation always hides a constant which, as a rule, does not depend on $n$ but may depend on dimension. With this notation, the above theorem can be formulated in the following way.

Theorem 3.2. For large enough $n$ and for every $K \in \mathcal{K}_{1}$,

$$
\operatorname{vol} K(1 / n) \ll \mathbb{E}(K, n) \ll \operatorname{vol} K(1 / n) \text {. }
$$

The content of Theorem 3.2 is that, instead of determining $\mathbb{E}(K, n)$, one can determine the volume of the wet part (which is usually simpler) and obtain the order of magnitude of $\mathbb{E}(K, n)$. The reader will have no difficulty understanding that for the unit ball $B^{d}$ in $\mathbb{R}^{d}$ the wet part $B^{d}(v \leq t)$ is the annulus $B^{d} \backslash(1-h) B^{d}$ where $h$ is of order $t^{2 /(d+1)}$. Thus

$$
\mathbb{E}\left(B^{d}, n\right) \approx \operatorname{vol} B^{d}(1 / n) \approx n^{-2 /(d+1)} .
$$

Similarly, for the unit cube $Q^{d}$ in $\mathbb{R}^{d}$ the floating body with parameter $t$ (in the subcube $\left.[0,1 / 2]^{d}\right)$ is bounded by the hypersurface $\left\{x \in \mathbb{R}^{d}: \prod x_{i}=d^{d} t / d !\right\}$. 
From this, the volume of the wet part can be determined easily (see also Section 12),

$$
\mathbb{E}\left(Q^{d}, n\right) \approx \operatorname{vol} Q^{d}(1 / n) \approx \frac{(\ln n)^{d-1}}{n} .
$$

This is the second level of explanation for the very different behaviour of $\mathbb{E}(K, n)$ : the volume of the wet part varies heavily depending on the boundary structure of $K$.

\section{The Volume of the Wet Part}

By the theorems of the previous section, the order of magnitude of $\mathbb{E}(K, n)$ is determined by that of vol $K(1 / n)$. In this section we state several results on the function $t \mapsto \operatorname{vol} K(t)$. In particular, we are interested in the cases when this function is maximal and minimal.

The wet part $K(t)=K(v \leq t)$ is a kind of inner parallel body to the boundary of $K$. We note first that the function $v: K \rightarrow \mathbb{R}$ is invariant (or rather equivariant) under non-degenerate linear transformations $A: \mathbb{R}^{d} \rightarrow \mathbb{R}^{d}$. Precisely, recalling the notation $v(x)=v_{K}(x)$, we have

$$
v_{A K}(A x)=|\operatorname{det} A| v_{K}(x)
$$

since $C_{A K}(A x)=A\left(C_{K}(x)\right)$. This also shows that the quantity

$$
\frac{\operatorname{vol} K(v \leq t \operatorname{vol} K)}{\operatorname{vol} K}
$$

is invariant under non-degenerate linear transformations.

Theorem 4.1. Assume $K \in \mathcal{K}_{1}$ and $t \geq 0$. Then

$$
\operatorname{vol} K(t) \gg t\left(\ln \frac{1}{t}\right)^{d-1} \text {. }
$$

This theorem is best possible (apart from the implied constant) as shown by polytopes. We need the following definition. A tower of a polytope $P$ is a chain of faces $F_{0} \subset F_{1} \subset \ldots, \subset F_{d-1}$ where $F_{i}$ is $i$-dimensional. Write $T(P)$ for the number of towers of $P$.

Theorem 4.2. Assume $P \in \mathcal{K}_{1}$ and $t \geq 0$. Then

$$
\operatorname{vol} P(t)=\frac{T(P)}{d^{d-1} d !} t\left(\ln \frac{1}{t}\right)^{d-1}(1+o(1)) \text {. }
$$

The result is due to Schütt [50], and independently to Bárány and Buchta [9]. It is used in the proof of the following theorem. 
Theorem 4.3. Assume $P \in \mathcal{K}_{1}$ and $t \geq 0$. Then

$$
\mathbb{E}(P, n)=\frac{T(P)}{(d+1)^{d-1}(d-1) !} \frac{(\ln n)^{d-1}}{n}(1+o(1)) .
$$

This is a difficult theorem whose proof is based on work of Affentranger and Wieacker [1] and Bárány and Buchta [9]. Here we will only prove Theorem 4.2 in the simpler form saying that

$$
\operatorname{vol} P(t) \ll T(P) t\left(\ln \frac{1}{t}\right)^{d-1},
$$

where the implied constant depends on dimension only.

Concerning the upper bound on the volume of the wet part, or on $\mathbb{E}(K, n)$, the following result of Groemer [22] gives a complete answer.

Theorem 4.4. Among all convex bodies in $\mathcal{K}_{1}, \mathbb{E}(K, n)$ is maximal for ellipsoids, and only for ellipsoids.

The affine isoperimetric inequality (cf. Blaschke [14] and Schütt [52]) expresses a similar extremal property of ellipsoids.

Theorem 4.5. For all convex bodies in $\mathcal{K}_{1}$,

$$
\limsup _{t \rightarrow 0} t^{-\frac{2}{d+1}} \operatorname{vol} K(t)
$$

is maximal for ellipsoids, and only for ellipsoids.

In case of smooth convex bodies in $\mathbb{R}^{d}$ more precise information is available.

Theorem 4.6. For a convex body $K \in \mathcal{K}_{1}$ with $\mathcal{C}^{2}$ boundary and positive curvature $\kappa$ at each point of bd $K$,

$$
\mathbb{E}(K, n)=c(d)\left(\int_{\mathrm{bd} K} \kappa^{\frac{1}{d+1}} \mathrm{~d} S\right) n^{-\frac{2}{d+1}}(1+o(1)),
$$

where $\mathrm{d} S$ denotes integration over bd $K$.

The above results show that one can determine $\mathbb{E}(K, n)$ and vol $K(t)$ for smooth convex bodies and for polytopes. What happens between these two extreme classes of convex bodies is not a mystery: it is the usual unpredictable behaviour. Using the above results and a general theorem of Gruber [24] one can show the following.

Theorem 4.7. Assume $\omega(n) \rightarrow 0$ and $\Omega(n) \rightarrow \infty$. Then for most (in the Baire category sense) convex bodies in $\mathcal{K}_{1}$ one has, for infinitely many $n$,

$$
\mathbb{E}(K, n) \geq \omega(n) n^{-\frac{2}{d+1}},
$$

and also, for infinitely many $n$,

$$
\mathbb{E}(K, n) \leq \Omega(n) \frac{(\ln n)^{d-1}}{n} .
$$


There is, of course, an analogous theorem for $K(t)$ with $\omega(t)$ and $\Omega(t)$ whose formulation and proof are left to the interested reader.

We will only prove Theorem 4.1 and inequality (4).

\section{The Economic Cap Covering Theorem}

Everything interesting that can happen to a convex body happens near its boundary. The technique of cap coverings and $M$-regions is a powerful method to deal with the boundary structure of convex bodies. The proof of the economic cap covering theorem (see [10] and [4]) is based on this technique. It says the following.

Theorem 5.1. Assume $K \in \mathcal{K}_{1}$ and $0<\varepsilon<\varepsilon_{0}=(2 d)^{-2 d}$. Then there are caps $C_{1}, \ldots, C_{m}$ and pairwise disjoint convex sets $C_{i}^{\prime}, \ldots, C_{m}^{\prime}$ such that $C_{i}^{\prime} \subset C_{i}$, for each $i$, and

(i) $\bigcup_{1}^{m} C_{i}^{\prime} \subset K(\varepsilon) \subset \bigcup_{1}^{m} C_{i}$,

(ii) $\operatorname{vol} C_{i}^{\prime} \gg \varepsilon$ and $\operatorname{vol} C_{i} \ll \varepsilon$ for each $i$,

(iii) for each cap $C$ with $C \cap K(v>\varepsilon)=\emptyset$ there is a $C_{i}$ containing $C$.

The meaning is that the caps $C_{i}$ cover the wet part, but do not "over cover" it. In particular,

$$
m \varepsilon \ll \operatorname{vol} K(\varepsilon) \ll m \varepsilon .
$$

The next corollary expresses a certain concavity property of the function $\varepsilon \mapsto \operatorname{vol} K(\varepsilon)$. It says that, apart from the constant implied by the $\gg$ notation, the $d$ th root of $\operatorname{vol} K(\varepsilon)$ is a concave function. This will be sufficient for our purposes, that is, for the proof of Theorem 3.2.

Corollary 5.1. If $K \in \mathcal{K}_{1}, \varepsilon \leq \varepsilon_{0}$, and $\lambda \geq 1$, then

$$
\operatorname{vol} K(\varepsilon) \gg \lambda^{-d} \operatorname{vol} K(\lambda \varepsilon) \text {. }
$$

The proof of the above results relies heavily on the Macbeath regions and their properties. They are defined, with their properties explained, in the next section.

\section{Macbeath Regions}

Macbeath regions, or $M$-regions, for short, were introduced in 1952 by A. M. Macbeath [34]: given a convex body $K \in \mathcal{K}^{d}$, and a point $x \in K$, the corresponding $M$-region is, by definition,

$$
M(x)=M_{K}(x)=K \cap(2 x-K) .
$$


So $M(x)$ is, again, a convex set. It is centrally symmetric with centre $x$. We define the blown-up version of the $M$-region as follows

$$
M(x, \lambda)=M_{K}(x, \lambda)=x+\lambda[(K-x) \cap(x-K)] .
$$

This is just a blown-up copy of $M(x)$ from its centre $x$ with scalar $\lambda>0$.

We define the function $u: K \rightarrow R$ by

$$
u(x)=\operatorname{vol} M(x) .
$$

The level sets of $u$ are defined the same way as those of $v$,

$$
K(u \leq t)=\{x \in K: u(x) \leq t\}, K(u \geq t)=\{x \in K: u(x) \geq t\} .
$$

We note that the function $u: K \rightarrow \mathbb{R}$, just like $v$, is invariant (or rather equivariant) under non-degenerate linear transformations $A: \mathbb{R}^{d} \rightarrow \mathbb{R}^{d}$. That is,

$$
u_{A K}(A x)=|\operatorname{det} A| u_{K}(x)
$$

since $M_{A K}(A x)=A\left(M_{K}(x)\right)$. This also shows that the quantity

$$
\frac{\operatorname{vol} K(u \leq t \operatorname{vol} K)}{\operatorname{vol} K}
$$

is invariant under non-degenerate linear transformations, cf. (2).

$M$-regions have an important property that can often be used with induction on dimension. Namely, assume $H$ is a hyperplane and $x \in K \cap H$. Then, as it is very easy to see,

$$
M_{K \cap H}(x)=M_{K}(x) \cap H .
$$

The convexity of $K(u \geq t)$ is not as simple as that of $K(v \geq t)$ and we state it as a separate lemma.

Lemma 6.1. The set $K(u \geq t)$ is convex.

Proof (cf. Macbeath [34]). We check first that $\frac{1}{2}(M(x)+M(y)) \subset M\left(\frac{1}{2}(x+y)\right)$. So assume $a \in M(x)$, that is $a \in K$ and $a \in 2 x-K$, or $a=2 x-k_{1}$ for some $k_{1} \in K$. Similarly $b \in M(y)$ implies $b \in K$ and $b=2 y-k_{2}$ for some $k_{2} \in K$. Then, by the convexity of $K,(a+b) / 2 \in K$ and

$$
\frac{a+b}{2}=x+y-\frac{k_{1}+k_{2}}{2} \in 2 \frac{x+y}{2}-K,
$$

implying the claim. Now the Brunn-Minkowski inequality [46] together with the containment $\frac{1}{2}(M(x)+M(y)) \subset M\left(\frac{1}{2}(x+y)\right)$ implies that the function $u^{1 / d}$ is concave. Thus, in particular, the level sets $K(u \geq t)$ are convex.

The computation of $u(x)$ is simpler than that of $v(x)$ since one does not have to minimize. It turns out that $v(x) \approx u(x)$, when $x$ is close to the boundary 
of $K$. A word of warning is in place here: closeness to the boundary is to be expressed equivariantly, that is, in terms of how small $v(x)$ or $u(x)$ is, as both $u$ and $v$ are affinely equivariant.

We now list several properties of these functions and their interrelations. The proofs are technical and will be given in the next section which can be skipped on first reading. In each one of these lemmas we assume that $K$ is a convex body in $\mathcal{K}_{1}$ and $\varepsilon_{0}=d^{-1} 3^{-d}$.

Lemma 6.2. If $x, y \in K$ and $M(x, 1 / 2) \cap M(y, 1 / 2) \neq \emptyset$, then

$$
M(y, 1) \subset M(x, 5) .
$$

Lemma 6.3. We have $u(x) \leq 2 v(x)$, for all $x \in K$.

Lemma 6.4. If $x \in K$ and $v(x) \leq \varepsilon_{0}$, then

$$
C(x) \subset M(x, 2 d) .
$$

Lemma 6.5. If $x \in K$ and $v(x) \leq \varepsilon_{0}$, then $v(x)<(2 d)^{d} u(x)$.

Lemma 6.6. If $x \in K$ and $u(x) \leq(3 d)^{-d} \varepsilon_{0}$, then $v(x)<(2 d)^{d} u(x)$.

Lemma 6.7. $K(v \geq \varepsilon)$ contains no line segment on its boundary.

Lemma 6.8. Assume $C$ is a cap of $K$ and $C \cap K(v \geq \varepsilon)=\{x\}$, a single point. If $\varepsilon<\varepsilon_{0}$, then $\operatorname{vol} C \leq d \varepsilon$ and

$$
C \subset M(x, 2 d)
$$

Lemma 6.9. Every $y \in K(\varepsilon)$ is contained in a minimal cap $C(x)$ with $\operatorname{vol} C(x)=\varepsilon$ and $x \in \operatorname{bd} K(v \geq \varepsilon)$.

Lemma 6.10. If $\varepsilon \leq \varepsilon_{0}$, then $K(v \leq \varepsilon) \subset K(u \leq 2 \varepsilon)$. If $\varepsilon \leq(2 d)^{-d} \varepsilon_{0}$, then $K(u \leq \varepsilon) \subset K\left(v \leq(2 d)^{d} \varepsilon\right)$.

The importance of these lemmas lies in the fact that they show $u \approx v$ near the boundary of $K$ in a strong sense. Namely, under the conditions of Lemma 6.4 the minimal cap is contained in a blown-up copy of the Macbeath region. On the other hand, "half" of the Macbeath region is contained in the minimal cap. Precisely, if $C=K \cap H(a \leq t)$ is a minimal cap, then

$$
M(x) \cap H(a \leq t) \subset C(x) .
$$

This shows that there is a two-way street between $C(x)$ and $M(x): C(x)$ can be replaced by $M(x)$ and $M(x)$ by $C(x)$ whenever it is more convenient to work with the other one.

Remark. Lemma 6.4 was proved first by Ewald, Larman, and Rogers in [21], they show $C \subset M(x, 3 d)$. The slightly better constant here is new and so is the proof (given in the next section) and it comes from an effort to use affine-invariant methods when the statement is affinely invariant. 


\section{Proofs of the Properties of the $M$-regions}

Proof of Lemma 6.2 (from the ground breaking paper by Ewald, Larman, Rogers [21]). Assume $a$ is the common point of $M(x, 1 / 2)$ and $M(y, 1 / 2)$. Then

$$
a=x+\frac{1}{2}\left(x-k_{1}\right)=y+\frac{1}{2}\left(k_{2}-y\right)
$$

for some $k_{1}, k_{2} \in K$ implying $y=3 x-k_{1}-k_{2}$. Suppose now that $b \in M(y, 1)$. Then $b \in K \subset x+5(K-x)$ clearly, and $b=y+\left(y-k_{3}\right)$ with some $k_{3} \in K$. Consequently

$$
\begin{aligned}
b & =2 y-k_{3}=6 x-2 k_{1}-2 k_{2}-k_{3} \\
& =x+5\left(x-\left[\frac{2}{5} k_{1}+\frac{2}{5} k_{2}+\frac{1}{5} k_{3}\right]\right) \in x+5(x-K) .
\end{aligned}
$$

Lemma 6.3 follows from (9). Lemma 6.4 is also from [21], the proof below is a slight improvement on the constant.

Proof of Lemma 6.4. The basic observation is that if $C(x)=K \cap H(a \leq t)$ is a minimal cap, then $x$ is the centre of gravity of the section $K \cap H(a=t)$. This can be checked by a routine variational argument. We first prove the following.

Claim 7.1 Assume $C(x)$ has width $w$, and $K$ contains a point $k$ in the hyperplane $H(a=t+2 w)$. Then $C(x) \subset M(x, 2 d)$.

Proof. Assume that, on the contrary, there is a point $z \in C(x)$ which is not in $M(x, 2 d)$. Then $z \notin x+2 d(x-K)$ implying

$$
z^{*}=x-\frac{1}{2 d}(z-x) \notin K .
$$

Let $L$ be the two-dimensional plane containing $x, k$ and $z$, then $z^{*} \in L$ as well, and our problem has become a simple planar computation. Fix a coordinate system to $L$ with $x$ lying at the origin and the hyperplane $H(a=t)$ intersecting $L$ in the $y$ axis. In this setting $z^{*}=-\frac{1}{2 d} z$. The line aff $\{k, z\}$, resp. aff $\left\{k, z^{*}\right\}$ intersects the $y$ axis at the points $u \in K$ (since $k, z \in K$ ) and $u^{*} \notin K$ (since $k \in K$ and $z^{*} \notin K$ ). As $x$ is the centre of gravity of the $(d-1)$-dimensional section, $(d-1)\left\|u^{*}\right\|>\|u\|$ must hold. Write $k=\left(k_{1}, k_{2}\right)$ and $z=\left(z_{1}, z_{2}\right)$; the conditions imply that $k_{1}=2 w$ and $z_{1} \in[-w, 0]$. It is not hard to check that

$$
\|u\|=\frac{\left|k_{1} z_{2}-z_{1} k_{2}\right|}{k_{1}-z_{1}} \text { and }\left\|u^{*}\right\|=\frac{\left|k_{1} z_{2}-z_{1} y_{2}\right|}{2 d k_{1}+z_{1}} .
$$

Then $(d-1)\left\|u^{*}\right\| \geq\|u\|$ implies $(d-1)\left(k_{1}-z_{1}\right) \geq 2 d k_{1}+z_{1}$ or $-d z_{1} \geq(d+1) k_{1}$ contradicting $k_{1}=2 w$ and $z_{1} \in[-w, 0]$. 
The rest of the proof is what I like to call trivial volume estimates. We show that if $C(x)=K \cap H(a \leq t)$ is a minimal cap of width $w$ and $v(x) \leq \varepsilon_{0}$, then the width of $K$ in direction $a$ is at least $3 w$. Assume the contrary and let

$$
A=\max \left\{\operatorname{vol}_{d-1}(K \cap H(a=\tau))\right\} .
$$

Then $1=\operatorname{vol} K \leq 3 w A$ and $v(x) \geq w A /\left(d 3^{d}\right)$ and so $v(x) \geq 1 /\left(d 3^{d}\right)$ contradicting $v(x) \leq \varepsilon_{0}$.

Lemma 6.5 follows immediately.

Proof of Lemma 6.6. Assume that $u(x) \leq(2 d)^{-d} \varepsilon_{0}$. Let $C(x)=K \cap H(a \leq t)$ be the minimal cap at $x$. Suppose its width is $w$. We show that the width of $K$ in direction $a$ is at least $3 w$. This implies the lemma via Lemma 6.4. Assume the contrary. With the same setting as in the previous proof one sees that $\operatorname{vol}_{d-1}(K \cap H(a=t)) \geq A / 3^{d-1}$. As $x$ is the centre of gravity of this section, the Löwner-John theorem implies that

$$
\operatorname{vol}_{d-1}(M(x) \cap H(a=t)) \geq \frac{A}{(3(d-1))^{d-1}}
$$

and $u(x) \geq 2 /(3 d)^{d}$ follows.

Proof of Lemma 6.7. Let $x, y \in \operatorname{bd} K(v \geq \varepsilon)$ and assume $z=\frac{1}{2}(x+y)$ is also in $\operatorname{bd} K(v \geq \varepsilon)$. Then there is a minimal cap $C(z)$ of volume $\varepsilon$. $C(z)$ cannot contain $x$ (or $y$ ) in its interior as otherwise a smaller "parallel" cap would contain $x$ (or $y$ ). Then $C(z)$ must contain both $x$ and $y$ in its bounding hyperplane. Then it is a minimal cap for both $x$ and $y$. But both $x$ and $y$ cannot be the centre of gravity of the section $K \cap H(a=t)$ at the same time unless $x=y$.

Proof of Lemma 6.8. Denote the set of outer normals to $K(v \geq \varepsilon)$ at $z \in$ bd $K(v \geq \varepsilon$ ) by $N(z)$. It is well known (see [41]) that as $K(v \geq \varepsilon)$ is a convex body, $N(z)$ coincides with the cone hull of its extreme rays.

For $b \in S^{d-1}$ define $C^{b}$ as the unique cap $C^{b}=K \cap H(b \leq t)$ such that $C^{b} \cap K(v \geq \varepsilon) \neq \emptyset$ but $C^{b} \cap \operatorname{int} K(v \geq \varepsilon)=\emptyset$.

We show first that if $b$ is the direction of an extreme ray of $N(z)$, then $\operatorname{vol} C^{b}=\varepsilon$. To prove this we use a classical result of Alexandrov (see [46]) stating that at almost every point $z$ on the boundary of a convex body the supporting hyperplane is unique. This shows that if $z \in \operatorname{bd} K(v \geq \varepsilon)$ is such a point then $N(z) \cap S^{d-1}$ is a unique vector, to be denoted by $b(z)$. In this case, of course, $\operatorname{vol} C^{b(z)}=\varepsilon$.

Notice next that $N(z)$ is the polar of the minimal cone whose apex is $z$ and which contains $K(v \geq \varepsilon)$ (see [41] again). So there is a vector $w \in S^{d-1}$ such that $w \cdot b=0$ and $w \cdot x<0$ for all $x \in N(z), x \neq \lambda b(\lambda>0)$ and such that there are points $z(t) \in \operatorname{bd} K(v \geq \varepsilon)$ for all small enough $t>0$ with

$$
\|(z(t)-z)-t w\|=o(t)
$$


as $t \rightarrow 0$. Choose now a subsequence $z_{k} \in \mathrm{bd} K(v \geq \varepsilon)$ very close to $z(1 / k)$ with unique tangent hyperplane to $K(v \geq \varepsilon)$ (using Alexandrov's theorem). We may assume that $\lim b\left(z_{k}\right)$ exists and equals $b_{0} \in S^{d-1}$. It is easily seen that $b_{0} \in N(z)$. Assume $b_{0} \neq b$. Then, since $b\left(z_{k}\right) \in N\left(z_{k}\right)$,

$$
0 \geq b\left(z_{k}\right) \cdot\left(y-z_{k}\right),
$$

for every $y \in K(v \geq \varepsilon)$. In particular, for $y=z$ we get

$$
0 \geq b\left(z_{k}\right) \cdot\left(y-z_{k}\right)=-\frac{1}{k} b\left(z_{k}\right) \cdot u-o(1 / k)>-\frac{1}{2 k} b\left(z_{k}\right) \cdot u-o(1 / k)>0,
$$

for large enough $k$, a contradiction proving $b_{0}=b$. The continuity of the map $b \longmapsto \operatorname{vol} C^{b}$ implies vol $C^{b}=\varepsilon$.

Now let $C=K \cap H(a \leq t)$ be the cap in the statement of the lemma. Then $-a \in N(x)$ and thus $-a$ is in the cone hull of extreme rays of $N(x)$. Thus, by Carathédory's theorem, $-a$ is in the cone hull of $b_{1}, \ldots, b_{d} \in S^{d-1}$, where each $b_{i}$ represents an extreme ray of $N(z)$. Then $C$ is contained in $\cup C^{b_{i}}$. This implies that $\operatorname{vol} C \leq d \operatorname{vol} C^{b_{i}}=d \varepsilon$. Also, each $C^{b_{i}}$ is a minimal cap, so by Lemma 6.4 it is contained in $M(x, 2 d)$. Consequently,

$$
C \subset \bigcup_{1}^{d} C^{b_{i}} \subset M(x, 2 d) .
$$

Proof of Lemma 6.9. The minimal cap $C(y)=K \cap H(a \leq t)$ is internally disjoint from the floating body $K(v \geq \varepsilon)$. Let $\tau$ be the maximal number with $H(a \leq \tau)$ internally disjoint from $K(v \geq \varepsilon)$. By Lemma 6.7 the cap $C=K \cap H(a \leq \tau)$ contains a unique point $x \in \operatorname{bd} K(v \geq \varepsilon)$. The proof of Lemma 6.8 gives that

$$
y \in C(y) \subset C \subset \bigcup_{1}^{d} C^{b_{i}}
$$

where each $C^{b_{i}}$ is a minimal cap.

Proof of Lemma 6.10. We prove the first inclusion by showing that $K(u>$ $2 \varepsilon) \subset K(v>\varepsilon)$. As both sets are convex, it suffices to see that $x \in \operatorname{bd} K(u>$ $2 \varepsilon)$ implies $v(x) \geq \varepsilon$. The condition says that $u(x)=2 \varepsilon$ and Lemma 6.3 gives $2 \varepsilon=u(x) \leq 2 v(x)$.

The proof of the second inclusion is similar, just Lemma 6.5 is needed.

Remark. Most of the results here come from [21], [10] and [4]. But as I mentioned at the end of the previous section, some of the proofs here are new.

\section{Proof of the Cap Covering Theorem}

We start with a definition. If a cap $C=K \cap H(a \leq t)$ has width $w$, then $H(a=t-w)$ is a supporting hyperplane to $K$. The centre of the cap is the 
centre of gravity of the set $K \cap H(a=t-w)$. The blown-up copy of $C$ from its centre by a factor $\lambda>0$ is denoted by $C^{\lambda}$. It is clear that $C^{\lambda}$ lies between hyperplanes $H(a=t-w)$ and $H(a=t-w+\lambda w)$, and convexity implies that

$$
K \cap H(a \leq t-w+\lambda w) \subset C^{\lambda},
$$

and so vol $K \cap H(a \leq t-w+\lambda w) \leq \lambda^{d} \operatorname{vol} C$.

Choose a system of points $x_{1}, \ldots, x_{m}$ on the boundary of the floating body $K(v \geq \varepsilon)$ which is maximal with respect to the property

$$
M\left(x_{i}, 1 / 2\right) \cap M\left(x_{j}, 1 / 2\right)=\emptyset,
$$

for each $i, j$ distinct. Such a maximal system is finite since the $M$-regions are pairwise disjoint, all of them are contained in $K$ and $\operatorname{vol} M\left(x_{i}, 1 / 2\right)=$ $2^{-d} u\left(x_{i}\right) \geq(6 d)^{-d} v(x)=(6 d)^{-d} \varepsilon$.

Claim 8.1 For each $y \in K(\varepsilon)$ there is an $x \in \operatorname{bd} K(v \geq \varepsilon)$ with $y \in M(x)$.

Proof. Assume this is false for some $y \in K(\varepsilon)$. So for each $x \in \operatorname{bd} K(v \geq \varepsilon)$, we have $y \notin M(x)=K \cap(2 x-K)$ implying that $2 x-y \notin K$, for each $x \in K(v \geq \varepsilon)$. In other words, a homothetic copy of $K[\varepsilon]$ blown up from $y$ by a factor of 2 is disjoint from $K$. Let $H(a \leq t)$ be the halfspace containing $K$ and disjoint from the homothetic copy, and let $H(a \leq \tau)$ be the parallel halfspace disjoint from $K[\varepsilon]$ with its bounding hyperplane tangent to $K[\varepsilon]$. The cap $K \cap H(a \leq \tau)$ has volume at most $d \varepsilon$ by Lemma 6.8. The width of $K \cap H(a \leq t)$ is at most twice the width of $K \cap H(a \leq \tau)$. Thus we have, using (10),

$$
1=\operatorname{vol} K=\operatorname{vol}(K \cap H(a \leq t)) \leq 2^{d} \operatorname{vol}(K \cap H(a \leq \tau)) \leq 2^{d} d \varepsilon,
$$

contradicting $\varepsilon \leq \varepsilon_{0}$.

It will be easy to see now that

$$
K(\varepsilon) \subset \bigcup_{1}^{m} M\left(x_{i}, 5\right) .
$$

Indeed, for each $y \in K(\varepsilon)$ there is an $x \in \mathrm{bd} K[\varepsilon]$ with $y \in M(x)$. By the maximality of the system $x_{1}, \ldots, x_{m}$, there is an $x_{i}$ with $M(x, 1 / 2) \cap M\left(x_{i}, 1 / 2\right) \neq$ $\emptyset$. Lemma 6.2 shows then that $y \in M\left(x_{i}, 5\right)$.

We have now a covering of $K(\varepsilon)$ with $M$-regions. We are going to turn it into a covering with caps. The minimal cap at $x_{i}$ is given by $C\left(x_{i}\right)=$ $K \cap H\left(a_{i} \leq t_{i}\right)$, let $w_{i}$ be its width. Define

$$
C_{i}^{\prime}=M\left(x_{i}, 1 / 2\right) \cap H\left(a_{i} \leq t_{i}\right) \text { and } C_{i}=K \cap H\left(a_{i} \leq t_{i}+5 w_{i}\right) .
$$

It is evident that the $C_{i}^{\prime}$ are pairwise disjoint convex sets, each contained in $C_{i}$ and $\operatorname{vol} C_{i}^{\prime} \geq \frac{1}{2} \operatorname{vol} M\left(x_{i}, \frac{1}{2}\right) \geq \frac{1}{2}(2 d)^{-d} \varepsilon$ by Lemma 6.5 . On the other hand, 
$M\left(x_{i}, 5\right)$ lies between hyperplanes $H\left(a_{i}=t_{i}-w_{i}\right)$ and $H\left(a_{i}=t_{i}+5 w_{i}\right)$ and so it is contained in $C_{i}$. Finally, (10) shows that $\operatorname{vol} C_{i} \leq 6^{d} \operatorname{vol} C\left(x_{i}\right)=6^{d} \varepsilon$.

So far this is the proof of (i) and (ii) of the theorem. We now show how one can enlarge $C_{i}$ to satisfy (iii).

This is quite simple. With the previous notation, take $C_{i}=K \cap H\left(a_{i} \leq\right.$ $\left.t_{i}+(10 d-1) w_{i}\right)$. The new $C_{i}$ satisfy (i) and (ii) and vol $C_{i} \ll \varepsilon$. Moreover, $M\left(x_{i}, 10 d\right) \subset C_{i}$.

Consider now a cap $C$, disjoint from $K(v>\varepsilon)$. We may assume that our $C$ is maximal in the sense that $C \cap K(v \geq \varepsilon)$ is nonempty. Then, by Lemma 6.7, the intersection $C \cap K(v \geq \varepsilon)$ is a single point, say $x$, and by Lemma 6.8

$$
C \subset M(x, 2 d) .
$$

By the maximality of the system $x_{1}, \ldots, x_{m}$, there is an $x_{i}$ with $M(x, 1) \subset$ $M\left(x_{i}, 5\right)$. We claim that $M(x, 2 d) \subset M\left(x_{i}, 10 d\right)$. This will prove what we need.

The claim follows from a more general statement.

Fact. Assume $A$ and $B$ are centrally symmetric convex sets with centre $a$ and $b$ respectively. If $B \subset A$ and $\lambda \geq 1$, then

$$
b+\lambda(B-b) \subset a+\lambda(A-a) .
$$

Proof. We may assume $a=0$. Let $c \in B$, we have to prove that $b+\lambda(c-b) \in$ $\lambda A$. $B$ is symmetric, so $2 b-c \in B \subset A$, and $A$ is symmetric, so $c-2 b \in A$. Also, $A$ is convex and $c \in B \subset A$, thus $(1 / 2)(c+(c-2 b))=c-b \in A$. Then $c \in A$ and $c-b \in A$ imply $\lambda c \in \lambda A$ and $\lambda(c-b) \in \lambda A$. But $b+\lambda(c-b)$ lies on the segment connecting $\lambda c$ and $\lambda(c-b)$,

$$
b+\lambda(b-c)=\frac{1}{\lambda}(\lambda c)+\left(1-\frac{1}{\lambda}\right) \lambda(c-b) \in A,
$$

proving the fact.

Proof of Corollary 5.1. Let $C_{1}, \ldots, C_{m}$ be the economic cap covering from Theorem 5.1. We will show that

$$
K(\lambda \varepsilon) \subset \bigcup_{1}^{m} C_{i}^{d \lambda} .
$$

This will prove what we want. 
Consider $x \in K(\lambda \varepsilon)$, we may assume $x \notin \bigcup C_{i}$. The minimal cap $C(x)=$ $K \cap H(a \leq t)$ has centre $z$ and width $w$. The segment $[x, z]$ intersects bd $K(v \geq$ $\varepsilon)$ at the point $y$, and let $y \in H\left(a=t-w^{\prime}\right)$ and set $t^{\prime}=w-w^{\prime}$. Now

$$
\begin{aligned}
\varepsilon=v(y) & \leq \operatorname{vol}\left(K \cap H\left(a \leq t-w^{\prime}\right)\right)=\int_{t-w}^{t-w^{\prime}} \operatorname{vol}_{d-1}(K \cap H(a=\tau)) \mathrm{d} \tau \\
& \leq t^{\prime} \max \left\{\operatorname{vol}_{d-1}\left(K \cap(H(a=\tau)): t-w \leq \tau \leq t-w^{\prime}\right\}\right. \\
& \leq t^{\prime} \max \left\{\operatorname{vol}_{d-1}(K \cap(H(a=\tau)): t-w \leq \tau \leq t\} .\right.
\end{aligned}
$$

On the other hand

$$
\begin{aligned}
\lambda \varepsilon \geq v(x) & =\operatorname{vol}(K \cap H(a \leq t)) \\
& \geq \frac{1}{d} t^{\prime} \max \left\{\operatorname{vol}_{d-1}(K \cap(H(a=\tau)): t-w \leq \tau \leq t\},\right.
\end{aligned}
$$

where the last inequality holds since the double cone whose base is the maximal section $K \cap H(a=\tau)$ is contained in $C(x)$. Now $t / t^{\prime}=\|z-x\| /\|z-y\|$ and we get

$$
\|z-x\| \leq d \lambda\|z-y\| .
$$

Consider now the cap $C_{i}=K \cap H\left(a_{i} \leq t_{i}\right)$ that contains $y$. Let $z_{i}$ be the centre of $C_{i}$ and write $y_{i}$ for the intersection of $\left[z_{i}, x\right] \cap H\left(a_{i}=t_{i}\right)$. The line aff $\{z, x\}$ intersects the hyperplanes $H\left(a_{i}=t_{i}\right), H\left(a_{i}=t_{i}-w_{i}\right)$ respectively at $y^{\prime}$ and $z^{\prime}$. It is easy to check that the points $z^{\prime}, z, y, y^{\prime}, x$ come in this order on aff $\{z, x\}$. Consequently,

$$
\frac{\left\|x-z_{i}\right\|}{\left\|y_{i}-z_{i}\right\|}=\frac{\left\|x-z^{\prime}\right\|}{\left\|y^{\prime}-z^{\prime}\right\|} \leq \frac{\|x-z\|+\left\|z-z^{\prime}\right\|}{\|y-z\|+\left\|z-z^{\prime}\right\|} \leq \frac{\|x-z\|}{\|y-z\|} \leq d \lambda .
$$

So indeed $x \in \bigcup_{1}^{m} C_{i}^{d \lambda}$.

\section{Auxiliary Lemmas from Probability}

We will need an upper and lower bound for the quantity $\mathbb{P}\left\{x \notin K_{n}\right\}$ where $x$ is a fixed point of $K$ and the random polytope $K_{n}$ varies. The lower bound is simple: if $C(x)$ is the minimal cap of $x$, then clearly

$$
\mathbb{P}\left\{x \notin K_{n}\right\} \geq \mathbb{P}\left\{X_{n} \cap C(x)=\emptyset\right\}=(1-v(x))^{n},
$$

where $X_{n}$ is the random sample of $n$ points from $K$ generating $K_{n}$.

We mention at once that this implies the lower bound in Theorem 3.2, or, what is the same, in Theorem 3.1. 
Proof (of the lower bound in Theorem 3.1). Using the above inequality we get, for all $t>0$ that

$$
\begin{aligned}
\mathbb{E}(K, n) & =\int_{K} \mathbb{P}\left\{x \notin K_{n}\right\} \mathrm{d} x \geq \int_{K}(1-v(x))^{n} \mathrm{~d} x \\
& \geq \int_{K(t)}(1-v(x))^{n} \mathrm{~d} x \geq \int_{K(t)}(1-t)^{n} \mathrm{~d} x \geq(1-t)^{n} \operatorname{vol} K(t) .
\end{aligned}
$$

Choosing here $t=1 / n$ gives the lower bound with $c_{1}=1 / 4$, for instance. Note that $c_{1}$ is universal, it does not depend on the dimension.

We need an upper bound on $\mathbb{P}\left\{x \notin K_{n}\right\}$,

$$
\mathbb{P}\left\{x \notin K_{n}\right\} \leq 2 \sum_{i=0}^{d-1}\left(\begin{array}{c}
n \\
i
\end{array}\right)\left(\frac{u(x)}{2}\right)^{i}\left(1-\frac{u(x)}{2}\right)^{n-i} .
$$

Proof. We are going to use the following equality which is due to Wendel [58]. Assume $M$ is an 0 -symmetric $d$-dimensional convex body, and let $X_{n}$ be a random sample of uniform, independent points from $M$. Then

$$
\mathbb{P}\left\{0 \notin \operatorname{conv} X_{n}\right\}=2^{-n+1} \sum_{i=0}^{d-1}\left(\begin{array}{c}
n-1 \\
i
\end{array}\right) .
$$

(I will give a proof of this result at the end of the section.)

Let $x \in K$ be fixed and define $N(x)=X_{n} \cap M(x)$. Setting $n(x)=|N(x)|$ we have

$$
\begin{aligned}
\mathbb{P}\left\{x \notin K_{n}\right\} & =\sum_{m=0}^{n} \mathbb{P}\left\{x \notin K_{n} \mid n(x)=m\right\} \mathbb{P}\{n(x)=m\} \\
& \leq \sum_{m=0}^{n} \mathbb{P}\{x \notin \operatorname{conv} N(x) \mid n(x)=m\} \mathbb{P}\{n(x)=m\} \\
& =2 \sum_{m=0}^{n} 2^{-m} \sum_{i=0}^{d-1}\left(\begin{array}{c}
m-1 \\
i
\end{array}\right) \mathbb{P}\{n(x)=m\} .
\end{aligned}
$$

We used Wendel's equality. $\mathbb{P}\{n(x)=m\}$ is a binomial distribution with parameter $u=u(x)$. Thus 


$$
\begin{aligned}
\mathbb{P}\left\{x \notin K_{n}\right\} & \leq 2 \sum_{m=0}^{n} 2^{-m} \sum_{i=0}^{d-1}\left(\begin{array}{c}
m-1 \\
i
\end{array}\right)\left(\begin{array}{c}
n \\
m
\end{array}\right) u^{m}(1-u)^{n-m} \\
& =2 \sum_{i=0}^{d-1} \sum_{m=0}^{n}\left(\begin{array}{c}
m-1 \\
i
\end{array}\right)\left(\begin{array}{c}
n \\
m
\end{array}\right)\left(\frac{u}{2}\right)^{m}(1-u)^{n-m} \\
& \leq 2 \sum_{i=0}^{d-1} \sum_{m=i+1}^{n}\left(\begin{array}{c}
m \\
i
\end{array}\right)\left(\begin{array}{c}
n \\
m
\end{array}\right)\left(\frac{u}{2}\right)^{m}(1-u)^{n-m} \\
& =2 \sum_{i=0}^{d-1}\left(\begin{array}{c}
n \\
i
\end{array}\right) \sum_{m=i}^{n}\left(\begin{array}{c}
n-i \\
m-i
\end{array}\right)\left(\frac{u}{2}\right)^{m}(1-u)^{n-m} \\
& =2 \sum_{i=0}^{d-1}\left(\begin{array}{c}
n \\
i
\end{array}\right) \sum_{k=0}^{n-i}\left(\begin{array}{c}
n-i \\
k
\end{array}\right)\left(\frac{u}{2}\right)^{k+i}(1-u)^{n-i-k} \\
& =2 \sum_{i=0}^{d-1}\left(\begin{array}{c}
n \\
i
\end{array}\right)\left(\frac{u}{2}\right)^{i}\left(1-\frac{u}{2}\right)^{n-i} \cdot
\end{aligned}
$$

Proof of Wendel's equality. We start with the following simple fact. Assume $H_{1}, \ldots, H_{n}$ are hyperplanes in $\mathbb{R}^{d}$ in general position that is, every $d$ of them has exactly one point in common and no $d+1$ of them intersect. The set $\mathbb{R}^{d} \backslash \cup_{1}^{n} H_{i}$ is the disjoint union of pairwise disjoint open sets, to be called cells. Each cell is a convex polyhedron.

Claim 9.1 The number of cells is exactly $\sum_{i=0}^{d}\left(\begin{array}{c}n \\ i\end{array}\right)$.

Proof. We prove this by induction on $d$. Everything is clear when $d=1$. Assume $d>1$ and the statement is true in $\mathbb{R}^{d-1}$. Let $a \in \mathbb{R}^{d}$ be a unit vector in general position and let $C$ be one of the cells. If $\min \{a \cdot x: x \in C\}$ is finite, then it is reached at a unique vertex of $C$ which is the intersection of some $d$ hyperplanes $H_{i_{1}}, \ldots, H_{i_{d}}$. There are $\left(\begin{array}{l}n \\ d\end{array}\right)$ such minima and each one comes from a different cell. So exactly $\left(\begin{array}{l}n \\ d\end{array}\right)$ cells have a finite minimum in direction $a$. Let $K$ be a number smaller than each of these $\left(\begin{array}{l}n \\ d\end{array}\right)$ minima. The rest of the cells are unbounded in this direction, so they all intersect the hyperplane $H$ with equation $a \cdot x=K$. The induction hypothesis can be used in $H$ (which is a copy of $\mathbb{R}^{d-1}$ ) to show that the number cells, unbounded in direction $a$ is $\sum_{i=0}^{d-1}\left(\begin{array}{c}n \\ i\end{array}\right)$. This finishes the proof of the claim.

Now for the proof of Wendel's equality. The basic observation is that choosing the points $x_{1}, \ldots, x_{n}$ and choosing the points $\varepsilon_{1} x_{1}, \ldots, \varepsilon_{n} x_{n}$ (where each $\varepsilon_{i}=$ \pm 1 ) is equally likely. So we want to see that, out of the $2^{n}$ such choices, how many will not have the origin in their convex hull. If $0 \notin\left[\varepsilon_{1} x_{1}, \ldots, \varepsilon_{n} x_{n}\right]$, then all the $\varepsilon_{i} x_{i}$ are contained in the open halfspace $\left\{x \in \mathbb{R}^{d}: a \cdot x>0\right\}$ for some unit vector $a \in \mathbb{R}^{d}$. The conditions $a \cdot\left(\varepsilon_{i} x_{i}\right)>0$ show that all halfspaces 
containing each $\varepsilon_{i} x_{i}\left(i=1, \ldots, n\right.$, the $\varepsilon_{i}$ are fixed) have their normal $a$ in the cone

$$
\bigcap_{1}^{n}\left\{y \in \mathbb{R}^{d}: y \cdot\left(\varepsilon_{i} x_{i}\right)>0\right\} .
$$

So the question is how many such cones there are. Or, to put it differently, when you delete the hyperplanes $H_{i}=\left\{y \in \mathbb{R}^{d}: y \cdot x_{i}=0\right\} i=1, \ldots, n$ from $\mathbb{R}^{d}$ you get pairwise disjoint open cones $C_{\alpha}$; how many such cones are there? Surprisingly, this number is independent of the position of the $x_{i}$ (if they are in general position and, in the given case, they are). We claim that this number is equal to

$$
2 \sum_{i=0}^{d-1}\left(\begin{array}{c}
n-1 \\
i
\end{array}\right) .
$$

This will, of course, prove Wendel's equality (14).

Consider now the hyperplane $H^{*}=\left\{y \in \mathbb{R}^{d}: y \cdot x_{n}=1\right\}$. The cones $C_{\alpha}$ come in pairs, $C_{\alpha}$ together with $-C_{\alpha}$ and only one of them intersects $H^{*}$. So the question is this. If you delete the hyperplanes $H_{i}, i=1, \ldots, n-1$ from $H^{*}$, how many connected components are left? This is answered by Claim 9.1, there are exactly

$$
\sum_{i=0}^{d-1}\left(\begin{array}{c}
n-1 \\
i
\end{array}\right)
$$

such cells.

\section{Proof of Theorem 3.1}

We only have to prove the upper bound. We start with the integral representation of $\mathbb{E}(K, n)$ and use the upper bound from (13),

$$
\begin{aligned}
\mathbb{E}(K, n) & =\int_{K} \mathbb{P}\{x \notin K\} \mathrm{d} x \\
& \leq \int_{K} 2 \sum_{i=0}^{d-1}\left(\begin{array}{c}
n \\
i
\end{array}\right)\left(\frac{u(x)}{2}\right)^{i}\left(1-\frac{u(x)}{2}\right)^{n-i} \mathrm{~d} x \\
& \leq 2 \sum_{i=0}^{d-1}\left(\begin{array}{c}
n \\
i
\end{array}\right) \int_{K}\left(\frac{u(x)}{2}\right)^{i}\left(1-\frac{u(x)}{2}\right)^{n-i} \mathrm{~d} x .
\end{aligned}
$$

$K$ is the disjoint union of the sets $K_{\lambda}$, for $\lambda=1,2, \ldots, n$, where

$$
K_{\lambda}=K((\lambda-1) / n \leq u<\lambda / n) .
$$

We integrate separately on each $K_{\lambda}$ using that, on $K_{\lambda}, u(x)<\lambda /(2 n)$ and $1-u(x) / 2 \leq \exp \{-(\lambda-1) /(2 n)\}$. Thus 
$\int_{K_{\lambda}}\left(\frac{u(x)}{2}\right)^{i}\left(1-\frac{u(x)}{2}\right)^{n-i} \mathrm{~d} x \ll\left(\frac{\lambda}{2 n}\right)^{i} \exp \{-(\lambda-1) / 4\} \operatorname{vol} K(u \leq \lambda / n)$.

We continue the inequality for $\mathbb{E}(K, n)$,

$$
\begin{aligned}
\mathbb{E}(K, n) & \ll 2 \sum_{i=0}^{d-1}\left(\begin{array}{c}
n \\
i
\end{array}\right) \sum_{\lambda=1}^{n}\left(\frac{\lambda}{2 n}\right)^{i} \exp \{-(\lambda-1) / 4\} \operatorname{vol} K(u \leq \lambda / n) \\
& \ll \sum_{\lambda=1}^{n} \sum_{i=0}^{d-1}\left(\begin{array}{c}
n \\
i
\end{array}\right)\left(\frac{\lambda}{2 n}\right)^{i} \exp \{-(\lambda-1) / 4\} \operatorname{vol} K(u \leq \lambda / n) \\
& =\sum_{\lambda=1}^{\Lambda} . .+\sum_{\lambda=\Lambda+1}^{n} . .
\end{aligned}
$$

where $\Lambda=(2 d)^{-d} \varepsilon_{0} n=d^{-1}(6 d)^{-d} n$. Note that

$$
\left(\begin{array}{c}
n \\
i
\end{array}\right)\left(\frac{\lambda}{2 n}\right)^{i} \ll \lambda^{i}
$$

So we have, using Lemma 6.10 and Corollary 5.1,

$$
\begin{aligned}
\sum_{\lambda=1}^{\Lambda} . . & \ll \sum_{\lambda=1}^{\Lambda} d \lambda^{d-1} \exp \{-(\lambda-1) / 4\} \operatorname{vol} K\left(v \leq(2 d)^{d} \lambda / n\right) \\
& \ll \sum_{\lambda=1}^{\Lambda} \lambda^{d-1} \exp \{-(\lambda-1) / 4\} \lambda^{d} \operatorname{vol} K(v \leq 1 / n) \\
& \ll \operatorname{vol} K(v \leq 1 / n) .
\end{aligned}
$$

Estimating the second sum is simpler, since one can use the trivial inequality $\operatorname{vol} K(u \leq \lambda / n) \leq 1$ to get

$$
\begin{aligned}
\sum_{\Lambda+1}^{n} . . & \ll \sum_{\Lambda+1}^{n} \lambda^{d-1} \exp \{-(\lambda-1) / 4\} \operatorname{vol} K\left(v \leq(2 d)^{d} \lambda / n\right) \\
& \ll \sum_{\Lambda+1}^{n} \lambda^{d-1} \exp \{-(\lambda-1) / 4\} \\
& \ll \operatorname{vol} K(v \leq 1 / n) .
\end{aligned}
$$

Thus we have $\mathbb{E}(K, n) \ll \operatorname{vol} K(1 / n)$.

Remark. This proof comes from the paper [10].

\section{Proof of Theorem 4.1}

We start with introducing further notation. Fix $a \in S^{d-1}$ and let $H\left(a=t_{0}\right)$ be the hyperplane whose intersection with $K$ has maximal $(d-1)$-dimensional 
volume among all hyperplanes $H(a=t)$. Assume the width of $K$ in direction $a$ is at most $2 t_{0}$; if this were not the case we would take $-a$ instead of $a$. As $a$ will be fixed during this proof we simply write $H(t)=H(a=t)$. Assume further that $H(0)$ is the tangent hyperplane to $K$. Define

$$
Q(t)=H(t) \cap K \text { and } q(t)=\operatorname{vol}_{d-1} Q(t) .
$$

The choice of $t_{0}$ ensures that, for $t \in\left[0, t_{0}\right]$,

$$
q(t) \geq\left(\frac{t}{t_{0}}\right)^{d-1} q\left(t_{0}\right) \text { and } 2 t_{0} q\left(t_{0}\right) \geq \operatorname{vol} K=1 .
$$

Claim 11.1 For $\varepsilon>0$ and for $t \in\left[0, t_{0}\right]$,

$$
Q(t)\left(u_{Q(t)} \leq \varepsilon /(2 t)\right) \subset K\left(u_{K} \leq \varepsilon\right) \cap H(t) .
$$

Proof. We are going to show that $x \in H(t) \cap K$ implies $u_{K}(x) \leq 2 t u_{Q(t)}(x)$. This of course proves the lemma.

Notice first that $M(x)$ lies between hyperplanes $H(0)$ and $H(2 t)$. Thus

$$
u(x)=\int_{0}^{2 t} \operatorname{vol}_{d-1}(M(x) \cap H(\tau)) \mathrm{d} \tau \leq 2 t \operatorname{vol}_{d-1}(M(x) \cap H(t)),
$$

since $M(x)$ is centrally symmetric, so its largest section is the middle one. Observe next that

$$
M(x) \cap H(t)=M_{Q(t)}(x),
$$

which follows from (8). Consequently $u(x) \leq 2 t \mathrm{vol}_{d-1} M_{Q(t)}(x)=2 t u_{Q(t)}(x)$.

We show next that, for $\varepsilon \in[0,1]$,

$$
\operatorname{vol} K(u \leq \varepsilon) \gg \varepsilon\left(\ln \frac{1}{\varepsilon}\right)^{d-1} .
$$

Then Lemma 6.10 implies that, for $\varepsilon \leq(2 d)^{-2 d} \varepsilon_{0}$,

$$
\operatorname{vol} K(v \leq \varepsilon) \geq \operatorname{vol} K\left(u \leq(2 d)^{-d} \varepsilon\right) \gg \varepsilon\left(\ln \frac{1}{\varepsilon}\right)^{d-1} .
$$

When $\varepsilon \geq(2 d)^{-2 d}$ the statement of the theorem follows from the fact that $\varepsilon \mapsto \operatorname{vol} K(v \leq \varepsilon)$ is an increasing function of $\varepsilon$.

We prove (16) by induction on $d$. The case $d=1$ trivial. We will need the induction hypothesis in its invariant form (7): for $Q \in \mathcal{K}^{d-1}$ and for $\eta>0$

$$
\frac{\operatorname{vol} Q\left(u_{Q} \leq \eta \operatorname{vol} Q\right)}{\operatorname{vol} Q} \geq c_{d-1} \eta\left(\ln \frac{1}{\eta}\right)^{d-2} .
$$


We have

$$
\begin{aligned}
\operatorname{vol} K(u \leq \varepsilon) & \geq \operatorname{vol}(K(u \leq \varepsilon) \cap H(a \leq t)) \\
& =\int_{0}^{t_{0}} \operatorname{vol}_{d-1}(K(u \leq \varepsilon) \cap H(t)) \mathrm{d} t \\
& \geq \int_{0}^{t_{0}} \operatorname{vol}_{d-1} Q(t)\left(u_{Q(t)} \leq \varepsilon /(2 t)\right) \mathrm{d} t
\end{aligned}
$$

according to Claim 11.1. Define $\eta=\eta(t)=\varepsilon /(2 t q(t))$ and let $t_{1}$ be the unique solution to $\eta(t)=1$ between 0 and $t_{0}$. The induction hypothesis implies that for $t \in\left[t_{1}, t_{0}\right]$,

$$
\begin{aligned}
\operatorname{vol}_{d-1} Q(t)\left(u_{Q(t)} \leq \eta q(t)\right) & \geq c_{d-1} q(t) \eta\left(\ln \frac{1}{\eta}\right)^{d-2} \\
& =c_{d-1} \frac{\varepsilon}{2 t}\left(\ln \frac{2 t q(t)}{\varepsilon}\right)^{d-2} \\
& \geq c_{d-1} \frac{\varepsilon}{2 t}\left(\ln \left(\frac{2 t}{\varepsilon}\left(\frac{t}{t_{0}}\right)^{d-1}\right)\right)^{d-2},
\end{aligned}
$$

where the last inequality comes from (15). We continue with vol $K(u \leq \varepsilon)$,

$$
\operatorname{vol} K(u \leq \varepsilon) \geq \int_{t_{1}}^{t_{0}} c_{d-1} \frac{\varepsilon}{2 t}\left(\ln \left(\frac{2 t^{d} q\left(t_{0}\right)}{\varepsilon t_{0}^{d-1}}\right)\right)^{d-2} \mathrm{~d} t .
$$

Define $\alpha$ by $\alpha^{d}=2 q\left(t_{0}\right) /\left(\varepsilon t_{0}^{d-1}\right)$ and set $t_{2}=1 / \alpha$. In view of (15) again, $t_{1} \leq t_{2} \leq t_{0}$. Substitute now $\tau=\alpha t$ with $\tau_{i}=\alpha t_{i}, i=0,2$. We finally have

$$
\begin{aligned}
\operatorname{vol} K(u \leq \varepsilon) & \geq \int_{t_{1}}^{t_{0}} c_{d-1} \frac{\varepsilon}{2 \tau}(\ln \tau)^{d-2} \mathrm{~d} \tau \\
& =\frac{\varepsilon c_{d-1}}{2(d-1)}\left(\ln \frac{t_{0}\left(2 q\left(t_{0}\right)\right)^{1 / d}}{\left(\varepsilon t_{0}^{d-1}\right)^{1 / d}}\right)^{d-1} \geq \frac{\varepsilon c_{d-1}}{2(d-1)}\left(\frac{1}{d} \ln \frac{1}{\varepsilon}\right)^{d-1},
\end{aligned}
$$

where the last inequality follows from (15).

Remark. This is the only proof known for Theorem 4.1 and it comes from [10]. The best possible constant in the inequality probably goes with the simplex. Note that in the proof we made full use of the two-way street between minimal caps and $M$-regions.

\section{Proof of (4)}

This is a repetition of the previous computation, just the inequalities go the other direction. We need to know vol $\triangle(v \leq t)$, where $\triangle$ is the $d$-dimensional simplex. 
Lemma 12.1. For all $t \leq e^{-d+1}$,

$$
\frac{\operatorname{vol} \triangle(v \leq t \operatorname{vol} \triangle)}{\operatorname{vol} \triangle} \ll t\left(\ln \frac{1}{t}\right)^{d-1} .
$$

We remark that the function on the left hand side of this inequality increases with $t$ while the one on the right hand side increases on $\left[0, e^{-d+1}\right]$ and decreases afterwards. That is the reason for the condition $t \leq e^{-d+1}$.

Proof. We use induction on $d ; d=1$ is simple, $d=2$ needs a bit of special care and is left to the reader. We may assume that $\triangle$ is the regular simplex of volume 1 , as our inequality is in equivariant form. Let $w_{0}, \ldots, w_{d}$ be the vertices and $a_{i}$ the unit outer normals to the facet opposite to $w_{i}, i=0, \ldots, d$. Then $a_{i} \cdot w_{i}=h_{i}$ and $a_{i} \cdot w_{j}=h_{i}^{*}$ with $h_{i}^{*}>h_{i}$, and for every $x \in \triangle$ there is an $i$ with

$$
h_{i} \leq a_{i} \cdot x \leq h_{i}+\frac{d}{d+1}\left(h_{i}^{*}-h_{i}\right) .
$$

This is quite easy to check. Consequently,

$$
\operatorname{vol} \triangle(v \leq t) \leq \sum_{0}^{d} \operatorname{vol}_{d-1}\left\{x \in \triangle: v(x) \leq t, a_{i} \cdot x \leq h_{i}+\frac{d}{d+1}\left(h_{i}^{*}-h_{i}\right)\right\} .
$$

Each term in the last sum is the same, so we work with $i=0$ only. Assume $w_{0}=0$, then $h_{0}=0$ as well and we set $h^{*}=h_{0}^{*}$ and drop the subscript 0 . Define

$$
Q_{h}=\triangle \cap H(a=h),
$$

which is a regular and $(d-1)$-dimensional simplex. Note that

$$
\operatorname{vol} \triangle \cap H(a \leq h)=\frac{h}{d} \operatorname{vol}_{d-1} Q_{h}=\left(\frac{h}{h^{*}}\right)^{d}
$$

follows easily. If $x \in Q_{h}$ and $v(x) \leq t$ with minimal cap $C(x)$, then

$$
t \geq v(x) \geq \frac{1}{d} \min \left(h, h^{*}-h\right) \operatorname{vol}_{d-1} Q_{h} \cap C(x) \geq \frac{1}{d} \min \left(h, h^{*}-h\right) v_{Q_{h}}(x) .
$$

For $0 \leq h \leq h^{*} / 2$ the minimum is $h$, and for $h \geq h^{*} / 2$ it is at least $h^{*} /(d+1)$. Using this we can estimate now

$$
\begin{aligned}
\operatorname{vol} \triangle(v \leq t) \leq & (d+1) \int_{0}^{\frac{d}{d+1} h^{*}} \operatorname{vol}_{d-1}\left\{x \in Q_{h}: v(x)=h, a \cdot x=h\right\} \mathrm{d} h \\
\ll & \int_{0}^{h^{*} / 2} \operatorname{vol}_{d-1} Q_{h}\left(v_{Q_{h}} \leq t d / h\right) \mathrm{d} h+ \\
& +\int_{h^{*} / 2}^{\frac{d}{d+1} h^{*}} \operatorname{vol}_{d-1} Q_{h}\left(v_{Q_{h}} \leq t d(d+1) / h^{*}\right) \mathrm{d} h .
\end{aligned}
$$


The induction hypothesis gives us directly that the second integral is bounded by $\ll t(\ln 1 / t)^{d-2}$. The first integral is to be split at $h^{0}$ which is defined by

$$
e^{-d+1}=\frac{t d}{h^{0} \operatorname{vol}_{d-1} Q_{h^{0}}}=\left(\frac{h^{0}}{h^{*}}\right)^{d} .
$$

The integral below $h^{0}$ is smaller than vol $\triangle \cap H\left(a \leq h^{0}\right)=e^{d-1} t$. On the remaining interval the induction hypothesis gives

$$
\begin{aligned}
\int_{h^{0}}^{h^{*} / 2}[. .] & \leq \int_{h_{0}}^{h^{*} / 2} \frac{t d}{h}\left(\ln \frac{h \operatorname{vol}_{d-1} Q_{h}}{t d}\right)^{d-2} \mathrm{~d} h \\
& =\frac{t d}{d-1}\left[\left(\ln \frac{h^{*} \operatorname{vol}_{d-1} Q_{h^{*} / 2}}{2 t d}\right)^{d-1}-\left(\ln \frac{h^{0} \operatorname{vol}_{d-1} Q_{h^{0}}}{t d}\right)^{d-1}\right] \\
& \ll t\left(\ln \frac{1}{t}\right)^{d-1} .
\end{aligned}
$$

Remark. The last step of the proof can be used to show that most of $\triangle(v \leq t)$ is concentrated near the vertices of the simplex in the following sense;

$$
\operatorname{vol}\left[\triangle(v \leq t) \cap\left\{x \in \triangle: \ln \frac{1}{t} \leq a x \leq \frac{d}{d+1} h^{*}\right\}\right] \leq t\left(\ln \frac{1}{t}\right)^{d-2} \ln \ln \frac{1}{t} .
$$

The proof is straightforward.

Now we turn to the proof of inequality (4).

We triangulate first the polytope $P$ by simplices $\triangle_{i}$ using vertices of $P$ only. Clearly, if $v(x) \leq t$, then $v_{\triangle_{i}}(x) \leq t$ for the simplex containing $x$. Consequently

$$
\begin{aligned}
\operatorname{vol} P(v \leq t) & \leq \sum \operatorname{vol} \triangle_{i}\left(v_{\triangle_{i}} \leq t\right) \leq \sum t\left(\ln \frac{\operatorname{vol} \triangle_{i}}{t}\right)^{d-1} \\
& \ll t\left(\ln \frac{1}{t}\right)^{d-1} .
\end{aligned}
$$

The implied constant turns out to be proportional to the number of simplices needed for the triangulation. The argument for the slightly better constant in (4) goes as follows. The last remark gives that $P(v \leq t)$ is concentrated near the vertices of $P$. Assume 0 is a vertex of $P$ and $H(a \leq 0)$ is a halfspace (with outer unit normal $a$ ) intersecting $P$ only at 0 . One shows first that, with $\tau=\ln \frac{1}{t}$

$$
P(v \leq t) \cap H(a \leq \tau) \ll t\left(\ln \frac{1}{t}\right)^{d-1},
$$

where the implied constant is proportional to the number of towers of the section $P \cap H(a=h), h$ very small but positive. This can be proved by 
induction on $d$ using the same integration on the sections $P \cap H(a=h)$ as in the proof of Lemma 12.1. The number of towers of this section is the number of towers of $P$ incident to the vertex 0 . Summing over all vertices gives the required constant.

Remark. These proofs are from [10] and [4].

\section{Expectation of $f_{k}\left(K_{n}\right)$}

The following simple identity is due to Efron [20]; for $K \in \mathcal{K}_{1}$,

$$
\mathbb{E} f_{0}\left(K_{n}\right)=n \mathbb{E}(K, n-1) .
$$

The proof is straightforward,

$$
\begin{aligned}
\mathbb{E} f_{0}\left(K_{n}\right) & =\sum_{i=1}^{n} \mathbb{P}\left\{x_{i} \text { is a vertex of } K_{n}\right\} \\
& =n \mathbb{P}\left\{x_{1} \text { is a vertex of } K_{n}\right\}=n \mathbb{P}\left\{x_{1} \notin\left[x_{2}, \ldots, x_{n}\right]\right\} \\
& =n \mathbb{P}\left\{x \notin K_{n-1}\right\}=n \mathbb{E}(K, n-1),
\end{aligned}
$$

where the last probability is taken with both $K_{n-1}$ and $x$ varying.

Theorem 3.2 determines then the order of magnitude of $\mathbb{E} f_{0}\left(K_{n}\right)$ as well. The expectation of $f_{k}\left(K_{n}\right)$, for $k=1, \ldots, d-1$, must be close to that of $\mathbb{E} f_{0}\left(K_{n}\right)$ since, as $n$ goes to infinity, $K_{n}$ looks locally like a "random" triangulation of $\mathbb{R}^{d-1}$ where you don't expect vertices of high degree. We have the following theorem from [4].

Theorem 13.1. For large enough $n$, for all $K \in \mathcal{K}_{1}$ and for all $k=$ $0,1, \ldots, d-1$,

$$
n \operatorname{vol} K(1 / n) \ll \mathbb{E} f_{k}\left(K_{n}\right) \ll n \operatorname{vol} K(1 / n) .
$$

The lower bound in case $k=0$ follows from Efron's identity and the lower bound in Theorem 3.1. The following fact will simplify the proof of Theorem 13.1.

Lemma 13.1. For all $0 \leq i<j \leq d-1$,

$$
f_{i}\left(K_{n}\right) \leq\left(\begin{array}{c}
j+1 \\
i+1
\end{array}\right) f_{j}\left(K_{n}\right) .
$$

Proof. Almost surely $K_{n}$ is a simplicial polytope. Double counting the pairs $\left(F_{i}, F_{j}\right)$ where $F_{i}$ and $F_{j}$ are faces of dimension $i$ and $j$ of $K_{n}$ with $F_{i} \subset F_{j}$ we have 


$$
f_{i}\left(K_{n}\right)=\sum_{F_{i}} 1 \leq \sum_{\left(F_{i}, F_{j}\right)} 1 \leq\left(\begin{array}{l}
j+1 \\
i+1
\end{array}\right) f_{j}\left(K_{n}\right) .
$$

So we see that for the upper bound in Theorem 13.1 it suffices to show the following.

Lemma 13.2. For large enough $n$ and for all $K \in \mathcal{K}_{1}$,

$$
\mathbb{E} f_{d-1}\left(K_{n}\right) \ll n \operatorname{vol} K(1 / n) .
$$

For the proof of this lemma we need a corollary to the economic cap covering theorem. To state it, some preparation is necessary.

Assume $x_{1}, \ldots, x_{k} \in K$, set $L=\operatorname{aff}\left\{x_{1}, \ldots, x_{k}\right\}$ and define

$$
v(L)=\max \{v(x): x \in L\} .
$$

We write $K^{k}$ for the set of ordered $k$-tuples $\left(x_{1}, \ldots, x_{k}\right)$ with $x_{i} \in K$ for each $i$.

Corollary 13.1. If $K \in \mathcal{K}_{1}, k=1,2, \ldots, d$ and $\varepsilon \leq \varepsilon_{0}$, then

$$
\left\{\left(x_{1}, \ldots, x_{k}\right) \in K^{k}: v(L) \leq \varepsilon\right\} \subset \bigcup_{1}^{m}\left(C_{i}, \ldots, C_{i}\right),
$$

where $C_{1}, \ldots, C_{m}$ is the set of caps from Theorem 5.1.

Proof. This is where we use part (iii) of Theorem 5.1. If $v(L) \leq \varepsilon$, then $L$ and $K(v>\varepsilon)$ are disjoint. By separation, there is a halfspace $H$, containing $L$ which is disjoint from $K(v>\varepsilon)$. Then the cap $C=K \cap H$ is also disjoint from $K(v>\varepsilon)$. Clearly, $C$ contains $x_{1}, \ldots, x_{k}$. Consider now $C_{i}$ from the cap covering with $C \subset C_{i}$. It is evident that

$$
\left(x_{1}, \ldots, x_{k}\right) \in(C, \ldots, C) \subset\left(C_{i}, \ldots, C_{i}\right) .
$$

\section{Proof of Lemma 13.2}

We are going to use (1) when $\phi(F)$ is equal to one if $F=\left[x_{1}, \ldots, x_{d}\right]$ is a facet of $K_{n}$ and 0 otherwise. Recall that $V\left(x_{1}, \ldots, x_{d}\right)$ is the volume of the smaller cap cut off from $K$ by aff $\left\{x_{1}, \ldots, x_{d}\right\}$ which is a hyperplane with probability one. Now Theorem 2.1 says that

$$
\mathbb{E} f_{d-1}\left(K_{n}\right)=\left(\begin{array}{l}
n \\
d
\end{array}\right) \int_{K} \ldots \int_{K}\left[(1-V)^{n-d}+V^{n-d}\right] \mathrm{d} x_{1} \ldots \mathrm{d} x_{d} .
$$


We split the domain of integration into two parts: $K_{1}$ is the subset of $K^{d}$ where the function $V$ is smaller than $(c \ln n) / n$, and $K_{2}$ is where $V \geq(c \ln n) / n$. The constant $c$ will be specified soon. Clearly $V \leq 1 / 2$. The integrand over $K_{2}$ is estimated as follows:

$$
\begin{aligned}
(1-V)^{n-d}+V^{n-d} & \leq \exp \{-(n-d) V\}+2^{-(n-d)} \\
& \leq 2 \exp \{-(n-d)(c \ln n) / n\} \\
& =2 n^{-c(n-d) / n}
\end{aligned}
$$

which is smaller than $n^{-(d+1)}$ if $c$ is chosen large enough (depending only on $d)$. Then the contribution of the integral on $K_{2}$ to $\mathbb{E} f_{d-1}\left(K_{n}\right)$ is at most $1 / n$, so it is very small since, trivially, $\mathbb{E} f_{d-1}\left(K_{n}\right)$ is at least one.

Now let $h$ be an integer with $2^{-h} \leq(c \ln n) / n$. For each such $h$ let $\mathcal{M}_{h}$ be the collection of caps $\left\{C_{1}, \ldots, C_{m(h)}\right\}$ forming the economic cap covering from Theorem 5.1 with $\varepsilon=2^{-h}$.

Assume now that $\left(x_{1}, \ldots, x_{d}\right) \in K_{1}$. We will denote by $C\left(x_{1}, \ldots, x_{d}\right)$ the cap cut off from $K$ by the hyperplane aff $\left\{x_{1}, \ldots, x_{d}\right\}$, clearly vol $C\left(x_{1}, \ldots, x_{d}\right)$ $=V\left(x_{1}, \ldots, x_{d}\right)$. We associate with $\left(x_{1}, \ldots, x_{d}\right)$ the maximal $h$ such that, for some $C_{i} \in \mathcal{M}_{h}, C\left(x_{1}, \ldots, x_{d}\right) \subset C_{i}$. It follows that

$$
V\left(x_{1}, \ldots, x_{d}\right) \leq \operatorname{vol} C_{i} \ll 2^{-h}
$$

and, by the maximality of $h$,

$$
V\left(x_{1}, \ldots, x_{d}\right) \geq 2^{-h-1},
$$

since otherwise $C\left(x_{1}, \ldots, x_{d}\right)$ would be contained in a cap from $\mathcal{M}_{h+1}$.

For such an $\left(x_{1}, \ldots, x_{d}\right)$ we have

$(1-V)^{n-d}+V^{n-d} \leq 2(1-V)^{n-d} \leq 2\left(1-2^{-h-1}\right)^{n-d} \leq 2 \exp \left\{-(n-d) 2^{-h-1}\right\}$.

Now we integrate over $K_{1}$ by integrating each $\left(x_{1}, \ldots, x_{d}\right)$ on its associated $C_{i} \in \mathcal{M}_{h}$. In the expression (18) the integral on $C_{i} \in \mathcal{M}_{h}$ is bounded by

$$
2 \exp \left\{-(n-d) 2^{-h-1}\right\}\left(\operatorname{vol} C_{i}\right)^{d} \ll \exp \left\{-(n-d) 2^{-h-1}\right\}\left(2^{-h}\right)^{d},
$$

as all the $x_{i}$ come from $C_{i}$. Summing this for all $C_{i} \in \mathcal{M}_{h}$ and all $h \geq h_{0}$ where $h_{0}=\lfloor(c \ln n) / n\rfloor$ we get that

$$
\begin{aligned}
\mathbb{E} f_{d-1}\left(K_{n}\right) & \ll\left(\begin{array}{l}
n \\
d
\end{array}\right) \sum_{h_{0}}^{\infty} \sum_{C_{i} \in \mathcal{M}_{h}} \exp \left\{-(n-d) 2^{-h-1}\right\} 2^{-h d} \\
& \ll\left(\begin{array}{l}
n \\
d
\end{array}\right) \sum_{h_{0}}^{\infty} \exp \left\{-(n-d) 2^{-h+1}\right\} 2^{-h d}\left|\mathcal{M}_{h}\right| \\
& \ll\left(\begin{array}{l}
n \\
d
\end{array}\right) \sum_{h_{0}}^{\infty} \exp \left\{-(n-d) 2^{-h+1}\right\} 2^{-h(d-1)} \operatorname{vol} K\left(2^{-h}\right),
\end{aligned}
$$


where the last inequality follows from (5).

The rest of the proof is a direct computation using Corollary 5.1. We sum first for $h \geq h_{1}$ where $h_{1}$ is defined by $2^{-h_{1}} \leq 1 / n<2^{-h_{1}+1}$. The sum from $h_{1}$ to infinity is estimated via

$$
\begin{aligned}
\sum_{h_{1}}^{\infty} . . & \leq \sum_{h_{1}}^{\infty} \exp \left\{-(n-d) 2^{-h+1}\right\} 2^{-h(d-1)} \operatorname{vol} K(1 / n) \\
& \leq \operatorname{vol} K(1 / n) \sum_{h_{1}}^{\infty} 2^{-h(d-1)} \leq n^{-(d-1)} \operatorname{vol} K(1 / n) .
\end{aligned}
$$

When $h_{0} \leq h<h_{1}$, we set $h=h_{1}-k$, so $k$ runs from 1 to $k_{1}=\ln \ln n+\ln c$. Then we use Corollary 5.1 to show that

$$
\operatorname{vol} K\left(2^{-h}\right) \leq \operatorname{vol} K\left(2^{k} / n\right) \ll 2^{k d} \operatorname{vol} K(1 / n) .
$$

Thus

$$
\begin{aligned}
\sum_{h_{0}}^{h_{1}-1} . . & \ll \sum_{k=1}^{k_{1}} \exp \left\{-(n-d) 2^{-h_{1}+k-1}\right\} 2^{\left(-h_{1}+k\right)(d-1)} 2^{k d} \operatorname{vol} K(1 / n) \\
& \ll n^{-(d-1)} \operatorname{vol} K(1 / n) \sum_{k=1}^{k_{1}} \exp \left\{-(n-d) 2^{k} / n\right\} 2^{k(d-1)} 2^{k d} \\
& \ll n^{-(d-1)} \operatorname{vol} K(1 / n) \sum_{k=1}^{\infty} \exp \left\{-2^{k-1}+2 d k \ln 2\right\} \\
& \ll n^{-(d-1)} \operatorname{vol} K(1 / n) .
\end{aligned}
$$

Remark. This proof shows that $\mathbb{E} f_{d-1}\left(K_{n}\right) \ll \operatorname{vol} K(1 / n)$. Then $\mathbb{E} f_{0}\left(K_{n}\right) \ll$ $\operatorname{vol} K(1 / n)$ follows from Lemma 13.1. Efron's identity implies that $\mathbb{E} f_{0}\left(K_{n}\right) \approx$ $\mathbb{E}(K, n)$. Thus the proof of Lemma 13.2 is a new proof of the upper bound in Theorem 3.2. We mention further that the proof of $\mathbb{E} f_{d-1}\left(K_{n}\right) \ll \operatorname{vol} K(1 / n)$ presented here is new and uses the cap covering theorem in a different and apparently more effective way than the previous proof from [4].

\section{Further Results}

There is a huge number of papers devoted to random polytopes and this survey is too short to explain or even mention most of them. We only consider the random variables $f_{i}\left(K_{n}\right)$ and vol $K_{n}$, or rather $\operatorname{vol}\left(K \backslash K_{n}\right)$, although several other functionals of $K_{n}$ have been investigated, like surface area, mean width, and other intrinsic volumes, and the Hausdorff distance of $K$ and $K_{n}$. The interested reader should consult the survey papers [60], [49]. 
Also, other models of random polytopes have been thoroughly studied. The random sample may come from the normal distribution, or from the boundary of $K$. Here a recent result of Schütt and Werner [54] should be mentioned. In a long and intricate proof they show the precise asymptotic behaviour of $\operatorname{vol}\left(K \backslash K_{n}\right)$ when $K$ is a smooth convex body and the random points are chosen from the boundary of $K$ according to some probability distribution.

Many papers have been devoted to deriving precise asymptotic formulae for $\mathbb{E}(K, n)$ and $\mathbb{E} f_{i}\left(K_{n}\right)$ for special classes of convex bodies: for smooth convex bodies and polytopes. The starting point is usually the formula in Theorem 2.1: assuming $V \leq \frac{1}{2}$ the term $V^{n-d}$ is exponentially small and we obtain

$$
\mathbb{E} \phi\left(K_{n}\right)=\left(\begin{array}{l}
n \\
d
\end{array}\right) \int_{K} \ldots \int_{K}(1-V)^{n-d} \phi(F) \mathrm{d} x_{1} \ldots \mathrm{d} x_{d}+O\left(2^{-n}\right) .
$$

Here one can apply an integral transformation using the Blaschke-Petkantschin identity [43] and the integral becomes an integral over all hyperplanes $E$ meeting $K$;

$$
\mathbb{E} \phi\left(K_{n}\right)=C_{d}\left(\begin{array}{l}
n \\
d
\end{array}\right) \int(1-V)^{n-d} g_{K}(E) \mu(\mathrm{d} E)+O\left(2^{-n}\right),
$$

where $\mu(\mathrm{d} E)$ represents integration over the Grassmannian of hyperplanes and

$$
\begin{aligned}
& g_{K}(E)=(d-1) ! \int_{K \cap E} \ldots \int_{K \cap E} \phi\left(\left[x_{1}, \ldots, x_{d}\right]\right) \times \\
& \times \operatorname{vol}_{d-1}\left(\left[x_{1}, \ldots, x_{d}\right]\right) \mathrm{d} x_{1} \ldots \mathrm{d} x_{d}
\end{aligned}
$$

where the $\mathrm{d} x_{i}$ now denote integration in $E$. The main contribution in the integral above arises when $V$ is close to $1 / n$ and so it depends on the local boundary properties of $K$. This works when $K$ is smooth and $\phi=f_{d-1}$ and gives (see Raynaud [35] and Wieacker [59])

$$
\mathbb{E} f_{d-1}\left(K_{n}\right)=b_{d} \int_{\mathrm{bd} K} \kappa^{1 /(d+1)} d S n^{\frac{d-1}{d+1}}(1+o(1)),
$$

where $\kappa>0$ is the Gauss curvature and $b_{d}$ is a constant. The method does not quite work for $f_{0}\left(K_{n}\right)$ for general smooth convex bodies, but it does for the Euclidean ball [59], which can be used to establish the result

$$
\mathbb{E} f_{0}\left(K_{n}\right)=b_{d}^{\prime} \int_{\mathrm{bd} K} \kappa^{1 /(d+1)} \mathrm{d} S n^{\frac{d-1}{d+1}}(1+o(1))
$$

for smooth enough convex bodies, see Bárány [5] and Schütt [53]. This implies, via Efron's identity (17), a similar asymptotic formula for $\mathbb{E}(K, n)$.

The precise asymptotic formula for $f_{0}\left(K_{n}\right)$ and $\mathbb{E}(K, n)$ when $K$ is a polytope is given by Theorem 4.2 plus Efron's identity. 
Besides many results on the expectation of various functionals of $K_{n}$, very little has been known about the distribution of these functionals up to quite recently. A notable exception is Groeneboom's result [23] establishing a central limit theorem in the following from. For a polygon $P$ is the plane, the distribution of $f_{0}\left(P_{n}\right)$ is close to the normal. Precisely, if $P$ has $r$ vertices, then

$$
\frac{f_{0}\left(P_{n}\right)-\frac{2}{3} r \ln n}{\sqrt{\frac{10}{27} r \ln n}} \rightarrow \mathcal{N}(0,1)
$$

in distribution, where $\mathcal{N}(0,1)$ is the standard normal distribution. Further, Cabo and Groeneboom [18] proved, in a version suggested by Buchta [17], that

$$
\frac{\mathbb{E}(P, n)-\frac{2}{3} r \frac{\ln n}{n}}{\sqrt{\frac{28}{27} r \frac{\ln n}{n^{2}}}} \rightarrow \mathcal{N}(0,1),
$$

again in distribution. Groeneboom showed the central limit theorem for the case of the unit disk and $f_{0}$, with the variance evaluated numerically. Hsing [26] proved that

$$
\frac{\mathbb{E}\left(B^{2}, n\right)-c_{1} n^{-2 / 3}}{\sqrt{c_{2} n^{-5 / 3}}} \rightarrow \mathcal{N}(0,1)
$$

in distribution, again. The explicit constants $c_{1}$ and $c_{2}$ have been determined by Buchta [17]. The asymptotic distribution of the Hausdorff distance between a planar convex body $K$ and $K_{n}$ has been determined with high precision by Bräker, Hsing, and Bingham [16].

In a series of remarkable papers Reitzner [36], [37] has established an upper bound on the variance of the missed volume and $f_{i}\left(K_{n}\right)$ in the case of smooth convex bodies $K$ :

$$
\begin{aligned}
\operatorname{var} \operatorname{vol}\left(K \backslash K_{n}\right) & \leq c(K) n^{-(d+3) /(d+1)} \\
\operatorname{var} f_{i}\left(K_{n}\right) & \leq c(K) n^{(d-1) /(d+1)}
\end{aligned}
$$

where the constants $c(K)$ depend on $K$ and dimension only. These estimates imply a strong law of large numbers for the corresponding functionals. In a recent and very interesting paper [38] Reitzner has given a lower bound for the variance (smooth convex bodies) which are of the same order of magnitude as the upper bounds above. Using this he has been able to show that both the missed volume and the number of $i$-dimensional faces of $K_{n}$ satisfy the central limit theorem, a real breakthrough result in the theory of random polytopes. His argument is based on several ingredients: (1) a general central limit theorem of Rinott [40] where only partial independence of the random variables in question is required, (2) the right estimate for the variance, and (3) the precise comparison of random polytopes and polytopes obtained from a Poisson process $X(n)$ of intensity $n$ intersecting the smooth convex body $K$. 
These results have been extended to the case when $K$ is a polytope in $\mathbb{R}^{d}$ by Reitzner and myself [12]. Using geometric properties of polytopes and their $u$ and $v$ functions, combined with the cap-covering technique we could show that

$$
\begin{aligned}
\operatorname{var} \operatorname{vol}\left(K \backslash K_{n}\right) & \approx n^{-2}(\ln n)^{d-1} \\
\operatorname{var} f_{i}\left(K_{n}\right) & \approx(\ln n)^{d-1}
\end{aligned}
$$

The implied constants depend on the polytope $P$ in question. The central limit theorem, both for missed volume and $f_{i}$, follows the same way as in Reitzner's paper [37]. It is more difficult to measure the partial dependence of the underlying graph. These new results open the possibility for the central limit theorem for the missed volume of general convex bodies, not only smooth ones or for polytopes. I conjecture, for instance, that

$$
\operatorname{var} \operatorname{vol}\left(K \backslash K_{n}\right) \approx \frac{\operatorname{vol} K(1 / n)}{n}
$$

for all convex bodies with the implied constants depending only on dimension.

Almost at the same time and from a completely different and unexpected direction, strong concentration results for random polytopes have been proved by $\mathrm{Van} \mathrm{Vu}$ [57]. He uses a probabilistic and combinatorial technique which has been very powerful in other cases as well (cf. [56], [28]) for proving tail estimates. I state his result only for the random variable $Y_{n}=\operatorname{vol}\left(K \backslash K_{n}\right)$. The setting is this. Given a $K \in \mathcal{K}_{1}, \varepsilon>0$ small, and $x \in K$ with $v(x)<\varepsilon$, define $S_{x, \varepsilon}$ as the set of points $y \in K$ such that the segment $[x, y]$ is disjoint from $K(v \geq \varepsilon)$. So $S_{x . \varepsilon}$ is the union of all $\varepsilon$-caps containing $x$. Define $g(\varepsilon)=$ $\sup \left\{\operatorname{vol} S_{x, \varepsilon}: x \in K(\varepsilon)\right\}$ and let $A=3 g(\varepsilon)$ and $B=36 n g(\varepsilon)^{2} \operatorname{vol} K(v \leq \varepsilon)$. With this notation the following holds.

Theorem 15.1. There are positive constants $\alpha, c$ and $\varepsilon_{0}$ such that for every $K \in \mathcal{K}_{1}$ and for every $n$ and $\lambda$ satisfying $(\alpha \ln n) / n<\varepsilon<\varepsilon_{0}$ and $0<\lambda \leq$ $B /\left(4 A^{2}\right)=n \operatorname{vol} K(v \leq \varepsilon)$ we have

$$
\mathbb{P}\left\{\left|Y_{n}-\mathbb{E} Y_{n}\right| \geq \sqrt{\lambda B}\right\} \leq 2 \exp \{-\lambda / 4\}+\exp \{-c \varepsilon n\} .
$$

This is a very strong result implying, for instance, large deviation inequalities for $Y$ and good bounds on the centred moments of $Y$, or on how close $Y_{n}$ is to $\mathbb{E}\left(Y_{n}\right)$. In the particular case of smooth convex bodies, Vu strengthens the above inequality showing that $B$ can be chosen of the same order of magnitude as the variance of $Y_{n}$. The interested reader can learn a lot from Vu's excellent paper [57].

It should be mentioned that Calka and Schreiber [19] have recently proved a large deviation inequality for $f_{0}\left(K_{n}\right)$ in the case when $K$ is the unit ball. The exponent in their estimate is $n^{(d-1) /(3 d+5)}$, the same that $\mathrm{Vu}$ proves for smooth convex bodies. 


\section{Lattice Polytopes}

Originally, Macbeath [34] introduced $M$-regions in order to study integer points in convex bodies. He observed the following interesting fact. The integer convex hull of $K$ is defined as $\left[K \cap \mathbb{Z}^{d}\right]$, that is, the convex hull of the lattice points in $K$. If $z \in \mathbb{Z}^{d}$ is a vertex of the integer convex hull of $K$, then $u_{K}(z)<2^{d}$. This follows from Minkowski's classical theorem: $M_{K}(z)$ is a centrally symmetric convex body with centre $z \in \mathbb{Z}^{d}$. If it has volume at least $2^{d}$, then it contains another lattice point, say $y$, and by central symmetry, it also contains $2 z-y \in \mathbb{Z}^{d}$. But then $z$ is not a vertex of the integer convex hull because it is contained in the segment $[y, 2 z-y]$.

Macbeath mentions that $u_{K}(x)$ "is the most interesting function that one can associate with a convex set".

In 1963, G. E. Andrews [2] proved a remarkable theorem saying that a lattice polytope $P \subset \mathbb{R}^{d}$ of volume $V>0$ cannot have more than

$$
\text { const } V^{\frac{d-1}{d+1}}
$$

vertices or facets. Alternative proofs were later found by Arnol'd [3], Konyagin and Sevastyanov [31], Schmidt [44], Bárány and Vershik [13]. Here is yet another proof, from [11], based on the technique of $M$-regions. What we will present here is a sketch of the proof of

$$
f_{d-1}(P) \ll V^{\frac{d-1}{d+1}}
$$

since this is another application of the technique of $M$-regions and cap coverings.

We start the proof by fixing $\varepsilon=\left(2(10 d)^{d}(d+1) ! V\right)^{-1}$. Note that in this way $\varepsilon V<\varepsilon_{0}$. Let $F$ be a facet of $P$ and let $x_{F}$ be the point on bd $P(v \geq \varepsilon V)$ where the tangent hyperplane to $P(v \geq \varepsilon V)$ is parallel to $F$. According to Lemma 6.7, $x_{F}$ is unique. Let $C_{F}$ stand for the cap cut off from $K$ by the hyperplane parallel to $F$ and passing through $x_{F}$.

Lemma 16.1. For distinct facets $F$ and $G$ of $P$

$$
M\left(x_{F}, 1 / 2\right) \cap M\left(x_{G}, 1 / 2\right)=\emptyset .
$$

To see this assume this intersection is nonempty. Then, by Lemma 6.2, $M\left(x_{G}, 1\right) \subset M\left(x_{F}, 5\right)$. Further, Lemma 6.8 combined with (11) shows that

$$
G \subset C_{G} \subset M\left(x_{G}, 2 d\right) \subset M\left(x_{F}, 10 d\right),
$$

where the last containment is a simple consequence of $M\left(x_{G}, 1\right) \subset M\left(x_{F}, 5\right)$. Even simpler is

$$
F \subset C_{F} \subset M\left(x_{F}, 2 d\right) \subset M\left(x_{F}, 10 d\right)
$$

Now $M\left(x_{F}, 10 d\right)$ contains both facets $F$ and $G$ so it contains $d+1$ affinely independent lattice points. Thus its volume is at least $1 / d$ !. Then, using again Lemma 6.3, 


$$
\begin{aligned}
\frac{1}{d !} & \leq \operatorname{vol} M\left(x_{F}, 10 d\right)=(10 d)^{d} u\left(x_{F}\right) \leq(10 d)^{d} 2 v\left(x_{F}\right) \\
& \leq 2(10 d)^{d} \varepsilon V=\frac{1}{(d+1) !} .
\end{aligned}
$$

This is a contradiction (due to the choice of $\varepsilon$ ), finishing the proof.

So the half $M$-regions $M\left(x_{F}, 1 / 2\right)$, for all facets $F$, are pairwise disjoint. Their "half" $M\left(x_{F}, 1 / 2\right) \cap C(F)$ lies completely in $P(\varepsilon)$. Then, by Theorem 4.5 combined with (7),

$$
\sum_{F} \frac{1}{2} \operatorname{vol} M\left(x_{F}, 1 / 2\right) \leq \operatorname{vol} P(v \leq \varepsilon V) \ll \varepsilon^{\frac{2}{d+1}} V \ll V^{\frac{d-1}{d+1}},
$$

where the summation is taken over all facets $F$ of $P$. Now, again by Lemma 6.6 ,

$$
\operatorname{vol} M\left(x_{F}, 1 / 2\right)=2^{-d} u\left(x_{F}\right) \geq 2^{-d}(2 d)^{-d} v\left(x_{f}\right) \gg \varepsilon V \gg 1 .
$$

The last two formulae show that the number of facets of $P$ is $\ll V^{\frac{d-1}{d+1}}$.

We mention that this implies, via a trick of Andrews, the following slightly stronger theorem whose proof can be found in [11].

Theorem 16.1. For a lattice polytope $P \in \mathbb{R}^{d}$ with volume $V>0$

$$
T(P) \ll V^{\frac{d-1}{d+1}} .
$$

Remark. The result and the proof is originally from [10], and the presentation here is close to the one in [6]. The same applies to the next section.

\section{Approximation}

There are two types of problems in the theory of approximation of a $K \in \mathcal{K}$ by polytopes belonging to a certain class $\mathcal{P}$ of polytopes. The first type is asking for a lower bound, that is, a statement of the form: no polytope $P \in \mathcal{P}$ approximates $K$ better than some function of $K$ and $\mathcal{P}$. The second is asking for the existence of a polytope $P \in \mathcal{P}$ which approximates $K$ well, hopefully as well as the previous function. To be less vague, we consider inscribed polytopes only (that is $P \subset K$ ) and we measure approximation by the relative missed volume, that is, by

$$
\operatorname{appr}(K, P)=\frac{\operatorname{vol}(K \backslash P)}{\operatorname{vol} K} .
$$

In this section we show how the cap-covering technique can be used to attack both type of approximation problems. As expected, the results do not give precise constants but tell the right order of magnitude.

We start with the problem of the second type. C. Schütt [52] proved two very neat and general results. 
Theorem 17.1. Given $K \in \mathcal{K}_{1}$ and $t \in\left(0, t_{0}\right]$ (where $t_{0}$ depends only on the dimension), there is a polytope $P$ with $K(v \geq t) \subset P \subset K$ for which

$$
f_{0}(P) \ll \frac{\operatorname{vol} K(t)}{t} .
$$

Theorem 17.2. Given $K \in \mathcal{K}_{1}$ and $t \in\left(0, t_{0}\right]$ (where $t_{0}$ depends only on the dimension), there is a polytope $P$ with $K(v \geq t) \subset P \subset K$ for which

$$
f_{d-1}(P) \ll \frac{\operatorname{vol} K(t)}{t} .
$$

This means that $\operatorname{appr}(K, P) \leq \operatorname{vol} K(t)$ and the lost volume is " $t$ per vertex", and " $t$ per facet", respectively. We will see below that, for smooth bodies, this is the best possible order of magnitude. Schütt's proof of these theorems is direct and technical. Here I present a simple argument showing the power and efficacy of the cap covering method. Nevertheless, this argument gives weaker constants than Schütt's original theorem and does not extend to approximation by circumscribed polytopes (cf. [52]).

As the theorems are affinely invariant, we may assume that $K$ is in standard position, i.e., it is sandwiched between two balls, both centred at the origin with the radius of the larger at most $d$ times that of the smaller. For both theorems, start with setting $\tau=\lambda t$ (where $\lambda$ is a constant depending on $d$ only) and choose a system of points $\left\{x_{1}, \ldots, x_{m}\right\}$ from bd $K(v \geq \tau)$ maximal with respect to the property that the $M\left(x_{i}, 1 / 2\right)$ are pairwise disjoint. The economic cap covering argument shows that

$$
m \ll \frac{\operatorname{vol} K(\tau)}{\tau} \ll \frac{\operatorname{vol} K(t)}{t} .
$$

We start with the case of the facets which is simpler. Fix $\lambda=6^{-d}$. Let $C\left(x_{i}\right)$ be a minimal cap, and define

$$
P=K \backslash \cup_{1}^{m} C\left(x_{i}\right)^{6} .
$$

We will show that (1) no $z \in$ bd $K$ belongs to $P$, and (2) $K(v \geq t) \subset P$. This clearly suffices for the facet case.

To see (1), assume $z \in \mathrm{bd} K$, and let $z^{*} \in \mathrm{bd} K(v \geq \tau)$ be the point on the segment connecting $z$ and the origin (which is inside $K(v \geq \tau)$ if $t$ is small enough). Maximality implies the existence of $i$ with $M\left(z^{*}, 1 / 2\right) \cap M\left(x_{i}, 1 / 2\right) \neq$ $\emptyset$, and by Lemma 6.2, $M\left(z^{*}, 1\right) \subset M\left(x_{i}, 5\right)$. It is easy to see, using the standard position of $K$, that $z \in M\left(z^{*}, 1\right)$, and so

$$
z \in M\left(z^{*}, 1\right) \subset M\left(x_{i}, 5\right) \subset C\left(x_{i}\right)^{6} .
$$

To check that (2) also holds, we write

$$
\operatorname{vol} C\left(x_{i}\right)^{6} \leq 6^{d} \operatorname{vol} C\left(x_{i}\right)=6^{d} \tau=t,
$$


so $u(x) \leq t$ for every point $x$ cut off from $K$ by one of the caps $C\left(x_{i}\right)^{6}$.

For the proof of the vertex case set $\lambda=d^{-1} 6^{-d}$. Let $y_{i}$ be the intersection, with bd $K$, of the halfline through $x_{i}$ starting from the origin, and define

$$
P=\left[y_{1}, y_{2}, \ldots, y_{m}\right] .
$$

Clearly $P \subset K$. So we have to show $K(v \geq t) \subset P$. Assume the contrary, then there is a halfspace $H_{1}$ with $P \cap H_{1}=\emptyset$ whose bounding hyperplane is tangent to $K(v \geq t)$. Note that no $y_{i}$ is in $H_{1}$. Let $H_{2}$ be the halfspace whose bounding hyperplane is parallel to that of $H_{1}$, and which is tangent to $K(v \geq \tau)$ at the point $z$. Set $C_{j}=K \cap H_{j}, j=1,2$. Lemma 6.8 says that $\operatorname{vol} C_{2} \leq d \tau$.

By the maximality of the $x_{i}, M(z, 1 / 2)$ intersects some $M\left(x_{i}, 1 / 2\right)$ and so

$$
y_{i} \in M\left(x_{i}, 1\right) \subset M(z, 5) .
$$

Here $y_{i} \in M\left(x_{i}, 1\right)$ follows from the standard position of $K$. It is not hard to see that the cap $C_{2}^{6}$ contains $M(z, 5)$. Further, the cap $C_{2}^{6}$ is contained in the cap $C_{1}$ as their bounding hyperplanes are parallel and vol $C_{1} \geq t$ (by Lemma 6.8), while, by the same lemma, vol $C_{2}^{6} \leq 6^{d} \operatorname{vol} C_{2} \leq 6^{d} d \tau=t$. Thus $y_{i} \in C_{1} \subset H_{1}$, a contradiction.

We turn now to the first type of approximation question. We will consider here the family of all polytopes inscribed in $K$ with at most $n s$-dimensional faces. Denote this class of polytopes by $\mathcal{P}_{n}(K, s)$. The usual question of approximation by inscribed polytopes with at most $n$ vertices, the case $\mathcal{P}_{n}(K, 0)$ in our notation, is well understood, see [25]. Given a smooth enough convex body $K$, for every polytope in $\mathcal{P}_{n}(K, 0)$

$$
\operatorname{appr}(K, P) \leq c(d, K) n^{-\frac{2}{d-1}}(1+o(1)),
$$

as $n \rightarrow \infty$. In the other direction, there exist polytopes $P$ in $\mathcal{P}_{n}(K, 0)$ with

$$
\operatorname{appr}(K, P) \geq c(d, K) n^{-\frac{2}{d-1}}(1+o(1)) .
$$

Here even the constant, and its dependence on $K$ and $d$, are almost completely known. In the same paper, Gruber proves an asymptotic formula for circumscribed polytopes with at most $n$ facets, and in [33], Ludwig gives exact asymptotic formulae for the unrestricted case with $n$ vertices and $n$ facets, respectively. (Approximation is measured as the relative volume of the symmetric difference of $P$ and $K$.)

Is there a similar estimate for $\mathcal{P}_{n}(K, s)$ when $0<s<d-1$ ? Or a weaker one, giving the order of magnitude of $\operatorname{appr}(K, P)$ ? This unusual approximation question has come up in connection with the integer convex hull (cf. [11]).

Again, $M$-regions and cap coverings are going to help. I present the basic ideas of the proof in the case when $K=B^{d}$, the unit ball in $\mathbb{R}^{d}$. This extends without serious difficulty to convex bodies whose Gaussian curvature is 
bounded away from 0 and $\infty$. It should be mentioned that K. Böröczky Jr in [15] has worked out several other cases of this type, for instance, inscribed, circumscribed, and unrestricted polytopes with at most $n s$-dimensional faces (again when $0<s<d-1$ ). His approach is different: it is based on local quadratic approximation of the boundary and uses power diagrams. Here is the result for the unit ball.

Theorem 17.3. For every polytope $P \in \mathcal{P}_{n}\left(B^{d}, s\right)$, and for large $n$

$$
\operatorname{appr}\left(B^{d}, P\right) \gg n^{-\frac{2}{d-1}} .
$$

The proof below is based on an idea from [11] which is used there when $s=d-1$. This particular case, when $K=B^{d}$, was first proved by Rogers [42]. We mention that the theorem holds for smooth convex bodies, not only for the Euclidean ball. But the technique and the arguments are simpler and cleaner in the case of $B^{d}$. The interested reader will have no difficulty in extending the proof below to smooth convex bodies.

We may suppose that

$$
\operatorname{vol}\left(B^{d} \backslash P\right) \leq b_{1} n^{-\frac{2}{d-1}}
$$

for any particular constant $b_{1}$ of our choice ( $b_{1}$ depending on $d$ ), as otherwise there is nothing to prove. We assume further that $s \geq 1$.

Let $F_{1}, \ldots, F_{n}$ denote the $s$-dimensional faces of $P$ and let $x_{i}$ be the nearest point of $F_{i}$ to the origin. The minimal cap $C\left(x_{i}\right)$ has width $h_{i}$. It is not hard to check that $F_{i} \subset C\left(x_{i}\right)$. Also, $\operatorname{vol}\left(C\left(x_{i}\right) \backslash P\right) \geq \frac{1}{2} \operatorname{vol} C\left(x_{i}\right)$. This means that vol $C\left(x_{i}\right)$ must be small, and so $h_{i}$ must be small. Consequently, $\operatorname{vol} C\left(x_{i}\right) \approx h_{i}^{\frac{d+1}{2}}$, as a quick computation reveals.

Choose next a subsystem $\left\{x_{i_{1}}, \ldots, x_{i_{m}}\right\}$ from the $x_{i}$ which is, as we are used to it by now, maximal with respect to the property that the $M$-regions $M\left(x_{i_{j}}, 1 / 2\right)$ are pairwise disjoint. To have simple notation set $z_{j}=x_{i_{j}}$. By Lemma 6.2 , every $C\left(x_{i}\right)$ is contained in some $M\left(z_{j}, 5\right)$. So writing $V$ for the set of vertices of $P$ we clearly have

$$
V \subset \cup_{1}^{n} F_{i} \subset \cup_{1}^{n} C\left(z_{i}\right) \subset \cup_{1}^{m} M\left(z_{j}, 5\right) .
$$

Fix $\rho=b_{2} n^{-\frac{1}{d-1}}$, where $b_{2}$ is to be defined later. We want to show that the set $V+\rho B^{d}$ covers at most half of $S^{d-1}=\mathrm{bd} B^{d}$. We estimate the surface area of this set by that of $S^{d-1} \cap \cup_{1}^{m}\left(M\left(z_{j}, 5\right)+\rho B^{d}\right)$, which is clearly 


$$
\begin{aligned}
& \ll \sum_{1}^{m}\left(\rho+h_{j}^{\frac{1}{2}}\right)^{d-1}=\sum_{j=1}^{m} \sum_{k=0}^{d-1}\left(\begin{array}{c}
d-1 \\
k
\end{array}\right) h_{j}^{\frac{k}{2}} \rho^{d-1-k} \\
& =\sum_{k=0}^{d-1}\left(\begin{array}{c}
d-1 \\
k
\end{array}\right) \rho^{d-1-k}\left(\sum_{j=1}^{m} h_{j}^{\frac{k}{2}}\right) \\
& \leq \sum_{k=0}^{d-1}\left(\begin{array}{c}
d-1 \\
k
\end{array}\right) \rho^{d-1-k} m\left(\frac{1}{m} \sum_{j=1}^{m} h_{j}^{\frac{d+1}{2}}\right)^{\frac{k}{d+1}} \\
& =m\left(\rho+\left(\frac{1}{m} \sum_{1}^{m} h_{j}^{\frac{d+1}{2}}\right)^{\frac{1}{d+1}}\right)^{d-1}
\end{aligned}
$$

where we used the inequality between the $k$ th and $(d+1)$ st means.

We claim now that the last expression is smaller than half the surface area of $S^{d-1}$ if the constants $b_{1}, b_{2}$ are chosen suitably. Indeed, as $m \leq n$,

$$
\rho=b_{2} n^{-\frac{1}{d-1}} \leq b_{2} m^{-\frac{1}{d-1}}
$$

Next, as the $M\left(z_{j}, 1 / 2\right)$ are pairwise disjoint and one quarter of their volume is contained in $B^{d} \backslash P, \sum_{j=1}^{m} h_{j}^{\frac{d+1}{2}} \ll b_{1} n^{-\frac{2}{d-1}}$. This implies

$$
\left(\frac{1}{m} \sum h_{j}^{\frac{d+1}{2}}\right)^{\frac{1}{d+1}} \leq b_{1}^{\frac{1}{d+1}} m^{-\frac{1}{d-1}}
$$

We just proved that

$$
\begin{aligned}
V+\rho B^{d} & \ll m\left(\rho+\left(\frac{1}{m} \sum_{1}^{m} h_{j}^{\frac{d+1}{2}}\right)^{\frac{1}{d+1}}\right)^{d-1} \\
& \leq m\left(\left(b_{2}+b_{1}^{\frac{1}{d+1}}\right) m^{-\frac{1}{d-1}}\right)^{d-1}=\left(b_{2}+b_{1}^{\frac{1}{d+1}}\right)^{d-1}
\end{aligned}
$$

where the constant implied by $\ll$ depends only on $d$. So choosing $b_{1}$ and $b_{2}$ suitably we can assure that $V+\rho B^{d}$ misses at least half of $S^{d-1}$. Consequently,

$$
S^{d-1} \backslash\left(V+\frac{\rho}{2} B^{d}\right)
$$

contains many pairwise disjoint caps of radius $\rho / 2$. This is shown by a greedy algorithm: assume the centres $y_{p} \in S^{d-1} \backslash\left(V+\rho B^{d}\right)$ of these caps $C_{p}$ have been chosen for $p=1,2, \ldots, q$ and the caps are pairwise disjoint. The caps with centres $y_{p}$ and radius $\rho$ cover at most

$$
q \rho^{d-1} \operatorname{vol}{ }_{d-1} S^{d-1}
$$


of $S^{d-1}$. So as long as this is smaller than the surface area of

$$
S^{d-1} \backslash\left(V+\rho B^{d}\right),
$$

there is room to choose the next centre $y_{q+1}$. The algorithm produces as many as $\gg \rho^{-(d-1)} \gg n$ pairwise disjoint caps. They are all disjoint from $P$, so the volume missed by $P$ is

$$
\sum \operatorname{vol} C_{p} \gg n \rho^{d+1} \gg n^{-\frac{2}{d-1}},
$$

finishing the proof of Theorem 17.3.

\section{How It All Began: Segments on the Surface of $K$}

The technique of $M$-regions and cap coverings was invented by Ewald, Larman, and Rogers in their seminal paper [21]. Their aim was to answer a beautiful question of Vic Klee [29], [30]: "Can the boundary of a convex body contain segments in all directions?" (this is the title of [30].) After partial results by McMinn, Besicovitch, Pepe, and Grünbaum and Klee the following basic result was proved in [21].

Theorem 18.1. Let $S(K)$ denote the set of unit vectors $v \in \mathbb{R}^{d}$ such that the boundary of the convex body $K \subset \mathbb{R}^{d}$ contains a segment parallel with $v$. Then $S(K)$ has $\sigma$-finite $(d-2)$-dimensional Hausdorff measure.

For the proof they invent and develop the technique of cap covering. They prove Lemma 6.4 and Lemma 6.2 which is one of the key steps and lies at the core of the method. It is in this paper where the first economic cap covering is proved and used. The target is to cover the boundary (not the wet part) with caps that have the same width.

Lemma 18.1. Assume $K \in \mathcal{K}$ contains a ball of radius $r$ and is contained in a ball of radius $R$. Given a positive $\varepsilon \leq \varepsilon_{0}$, there are caps $C_{1}, \ldots, C_{m}$ with

(i) bd $B \subset \cup_{1}^{m} C_{i}$

(ii) the width of $C_{i}$ is between $2 \varepsilon$ and $36 d \varepsilon$,

(iii) $\sum_{1}^{m} \operatorname{vol} C_{i} \ll \varepsilon \operatorname{vol} K$.

Here $\varepsilon_{0}$ and the constant in $\ll$ depend on $d, r, R$ only.

Thus the caps $C_{i}$ constitute an "economic cap covering" of bd $K$ in the sense that each $C_{i}$ has minimal width $\approx \varepsilon$ and their total volume is $\ll \varepsilon \operatorname{vol} K$. We have seen several versions and strengthenings of this result. It is worth mentioning that the economic cap covering lemma is a relative of the Besicovitch covering theorem.

Theorem 18.1 says that, for every convex body, the set of exceptional directions is small. Precisely, it has $\sigma$-finite 1-codimensional Hausdorff measure. 
(Exceptional direction means here a direction contained in bd $K$.) That the exceptional set is small is important and useful in other cases as well, for instance, in integral geometry. It is shown in [21] that the set of exceptional $r$-flats has $\sigma$-finite 1-codimensional Hausdorff measure in the space of all $r$ flats. (Here $K \in \mathcal{K}$, of course, and an $r$-flat is exceptional if it intersects bd $K$ in a set of dimension $r$.)

The method of [21] was simplified by Zalgaller [61], and developed further by Ivanov [27], and Schneider [45], [48]. Ivanov shows that the union of all lines in $\mathbb{R}^{d}$ that meet bd $K$ in a segment has $\sigma$-finite $(d-1)$-dimensional Hausdorff measure. A consequence is that for almost all points $x \notin K$ the shadow boundary of $K$ under central projection from $x$ is sharp. (The reader will have no difficulty stating the analogous consequence of Theorem 18.1.) The following results of Schneider [45] and [48] have applications in integral geometry. The proof method is based on that of [21] but is much more involved.

Theorem 18.2. Let $K, K^{\prime} \in \mathcal{K}$. The set of all rotations $\rho \in S O(d)$ for which $K$ and $\rho K^{\prime}$ contain parallel segments lying in parallel supporting hyperplanes has $\sigma$-finite 1-codimensional Hausdorff measure.

Theorem 18.3. Let $K, K^{\prime} \in \mathcal{K}$. The set of all rigid motions $\rho$ for which $K$ and $\rho K^{\prime}$ have an exceptional common boundary point is of Haar measure zero.

Here a point $x$, common to bd $K$ and bd $K^{\prime}$, is exceptional if the normal cones to $K$ at $x$ and to $K^{\prime}$ at $x$ contain a common halfline.

Schneider gives a sketch of the proof of Theorem 18.1 in [47], and a full proof, containing Zalgaller's simplification, in his excellent book [46]. The interested reader is advised to consult these references.

\section{References}

1. Affentranger, F., Wieacker, J.A.: On the convex hull of uniform random points in a simple $d$-polytope. Discrete Comput. Geom., 6, 291-305 (1991)

2. Andrews, G.E.: A lower bound for the volumes of strictly convex bodies with many boundary points. Trans. Amer. Math. Soc., 106, 270-279 (1963)

3. Arnol'd, V.I.: Statistics of integral convex polytopes (in Russian). Funktsional. Anal. i Prilozhen., 14, 1-3 (1980). English translation: Funct. Anal. Appl., 14, 79-84 (1980)

4. Bárány, I.: Intrinsic volumes and $f$-vectors of random polytopes. Math. Ann., 285, 671-699 (1989)

5. Bárány, I.: Random polytopes in smooth convex bodies. Mathematika, 39, 81-92 (1992)

6. Bárány, I.: The technique of $M$-regions and cap-coverings: a survey. Rend. Circ. Mat. Palermo, Ser. II, Suppl., 65, 21-38 (1999)

7. Bárány, I.: Sylvester's question: the probability that $n$ points are in convex position. Ann. Probab., 27, 2020-2034 (1999)

8. Bárány, I.: A note on Sylvester's four-point problem., Studia Sci. Math. Hungar., 38, 73-77 (2001) 
9. Bárány, I., Buchta, Ch.: Random polytopes in a convex polytope, independence of shape, and concentration of vertices. Math. Ann., 297, 467-497 (1993)

10. Bárány, I., Larman, D.G.: Convex bodies, economic cap coverings, random polytopes. Mathematika, 35, 274-291 (1988)

11. Bárány, I,. Larman, D.G.: The convex hull of the integer points in a large ball. Math. Ann., 312, 167-181 (1998)

12. Bárány, I., Reitzner, M.: Central limit theorems for random polytopes in convex polytopes. Manuscript (2005)

13. Bárány, I., Vershik, A. M.: On the number of convex lattice polytopes. Geom. Funct. Anal., 2, 381-393 (1992)

14. Blaschke, W.: Affine Differentialgeometrie. Springer, Berlin (1923)

15. Böröczky, K. Jr: Polytopal approximation bounding the number of $k$-faces. J. Approx. Theory, 102, 263-285 (1999)

16. Bräker, H., Hsing, T., Bingham, N.H.: On the Hausdorff distance between a convex set and an interior random convex hull. Adv. in Appl. Probab., 30, 295-316 (1998)

17. Buchta, Ch.: An identity relating moments of functionals of convex hulls. Discrete Comput. Geom., 33, 125-142 (2005)

18. Cabo, A.J., Groeneboom, P.: Limit theorems for functionals of convex hulls. Probab. Theory Related Fields, 100, 31-55 (1994)

19. Calka, P., Schreiber, T.: Large deviation probabilities for the number of vertices of random polytopes in the ball. Ann. Probab., (to appear)

20. Efron, B.: The convex hull of a random set of points. Biometrika, 52, 331-343 (1965)

21. Ewald, G., Larman, D.G., Rogers, C.A.: The directions of the line segments and of the $r$-dimensional balls on the boundary of a convex body in Euclidean space. Mathematika, 17, 1-20 (1970)

22. Groemer, H.: On the mean value of the volume of a random polytope in a convex set. Arch. Math., 25, 86-90 (1974)

23. Groeneboom, P.: Limit theorems for convex hulls. Probab. Theory Related Fields, 79, 327-368 (1988)

24. Gruber, P.M.: In most cases approximation is irregular. Rend. Circ. Mat. Torino, 41, 19-33 (1983)

25. Gruber, P.M.: Asymptotic estimates for best and stepwise approximation of convex bodies II. Forum Math., 5, 521-538 (1993)

26. Hsing, T.: On the asymptotic distribution of the area outside a random convex hull in a disk. Ann. Appl. Probab., 4, 478-493 (1994)

27. Ivanov, B. A.: Exceptional directions for a convex body (in Russian). Mat. Zametki, 20, 365-371 (1976). English translation: Math. Notes 20, 763-765 (1976)

28. Kim, J.H., Vu, V.H.: Concentration of multivariate polynomials and its applications. Combinatorica, 20, 417-434 (2000)

29. Klee, V.: Research problem No. 5. Bull. Amer. Math. Soc., 62, 419 (1957)

30. Klee, V.: Can the boundary of a $d$-dimensional convex body contain segment in all directions? Amer. Math. Monthly, 76, 408-410 (1969)

31. Konyagin, S.B., Sevastyanov, S.V.: Estimation of the number of vertices of a convex integral polyhedron in terms of its volume (in Russian). Funktsional. Anal. i Prilozhen., 18, 13-15 (1984). English translation: Funct. Anal. Appl., 18, 11-13 (1984) 
32. Leichtweiss, K.: Affine Geometry of Convex Bodies. Barth, Heidelberg (1998)

33. Ludwig, M.: Asymptotic approximation of smooth convex bodies by general polytopes. Mathematika, 46, 103-125 (1999)

34. Macbeath, A.M.: A theorem on non-homogeneous lattices. Ann. of Math., 56, 269-293 (1952)

35. Raynaud, H.: Sur l'enveloppe convexe des nuages de points aléatoires dans $\mathbb{R}^{n}$. J. Appl. Probab., 7, 35-48 (1970)

36. Reitzner, M.: Random polytopes and the Efron-Stein jackknife inequality. Ann. Probab., 31, 2136-2166 (2003)

37. Reitzner, M.: The combinatorial structure of random polytopes. Adv. Math., 191, 178-208 (2005)

38. Reitzner, M.: Central limit theorems for random polytopes. Prob. Theory Appl., (to appear)

39. Rényi, A., Sulanke, R.: Über die konvexe Hülle von $n$ zufällig gewählten Punkten. Z. Wahrsch. Verw. Gebiete, 2, 75-84 (1963)

40. Rinott, Y.: On normal approximation rates for certain sums of dependent random variables. J. Comput. Appl. Math., 55, 135-143 (1994)

41. Rockafellar, T.R.: Convex Analysis. Princeton Univ. Press, Princeton, NJ (1970)

42. Rogers, C.A.: The volume of a polyhedron inscribed in a sphere. J. London Math. Soc., 28, 410-416 (1953)

43. Santaló, L.A.: Integral Geometry and Geometric Probability. Addison-Wesley, Reading, MA (1976)

44. Schmidt, W.: Integral points on surfaces and curves. Monatsh. Math., 99, 45-82 (1985)

45. Schneider, R.: Kinematic measures for sets of colliding convex bodies. Mathematika, 25, 1-12 (1978)

46. Schneider, R.: Convex Bodies: the Brunn-Minkowski Theory. Encyclopedia of Mathematics and Its Applications 44, Cambridge University Press, Cambridge (1993)

47. Schneider, R.: Measures in convex geometry. Rend. Istit. Mat. Univ. Trieste, Suppl., 29, 215-265 (1999)

48. Schneider, R.: Convex bodies in exceptional relative positions. J. London Math. Soc. (2), 60, 617-629 (1999)

49. Schneider, R.: Discrete aspects of stochastic geometry. In: Goodman, J.E., O'Rourke, J. (eds.) Handbook of Discrete and Computational Geometry. Second edition, Chapman \& Hall/CRC, Boca Raton, FL (2004)

50. Schütt, C.: The convex floating body and polyhedral approximation. Israel J. Math., 73, 65-77 (1991)

51. Schütt, C.: Random polytopes and affine surface area. Math. Nachr., 170, 227249 (1994)

52. Schütt, C.: Floating bodies, illumination bodies, and polytopal approximation. In: Ball, K. M. (ed.) Convex Geometric Analysis. Math. Sci. Res. Inst. Publ., 43, Cambridge (1999)

53. Schütt, C.: Random polytopes and affine surface area. Math. Nachr., 170, 227249 (1994)

54. Schütt, C., Werner, E.: Polytopes with vertices chosen randomly from the boundary of a convex body. Geometric aspects of functional analysis, Lecture Notes in Math., 1807, 241-422, Springer, Berlin (2003)

55. Sylvester, J.J.: Question 1491. Educational Times, London, April (1864) 
56. Vu, V.H.: Concentration of non-Lipschitz functions and applications. Random Structures Algorithms, 20, 262-316 (2002)

57. Vu, V.H.: Sharp concentration of random polytopes. Geom. Funct. Anal., (to appear)

58. Wendel, J.G.: A problem in geometric probability. Math. Scand., 11, 109-111 (1962)

59. Wieacker, J.A.: Einige Probleme der polyedrischen Approximation. Diplomarbeit, Albert-Ludwigs-Universität, Freiburg im Breisgau (1978)

60. W. Weil, J. A. Wieacker: Stochastic geometry. In: Gruber, P.M., Wills, J. (eds.) Handbook of Convex Geometry, North-Holland, Amsterdam (1993)

61. Zalgaller, V. A.: $k$-dimensional directions singular for a convex body $F$ in $R^{n}$ (in Russian). Zapiski naučn. Sem. Leningrad. Otd. mat. Inst. Steklov, 27, 67-72 (1972). English translation: J. Soviet Math., 3, 437-441 (1972) 